\title{
Branching Random Walks in Space-Time Random Environment: Survival Probability, Global and Local Growth Rates]
}

\author{
Francis Comets 2 and Nobuo Yoshidd 3
}

\begin{abstract}
We study the survival probability and the growth rate for branching random walks in random environment (BRWRE). The particles perform simple symmetric random walks on the $d$-dimensional integer lattice, while at each time unit, they split into independent copies according to time-space i.i.d. offspring distributions. The BRWRE is naturally associated with the directed polymers in random environment (DPRE), for which the quantity called the free energy is well studied. We discuss the survival probability (both global and local) for BRWRE and give a criterion for its positivity in terms of the free energy of the associated DPRE. We also show that the global growth rate for the number of particles in BRWRE is given by the free energy of the associated DPRE, though the local growth rate is given by the directional free energy.
\end{abstract}

Short Title. Branching Walks in Random Environment

Key words and phrases. Branching random walks, random environment, survival, global growth, local growth

AMS 2000 subject classifications. Primary 60K37; secondary 60F20, 60J80, 82D30, 82D60

\section{Contents}

1 Introduction

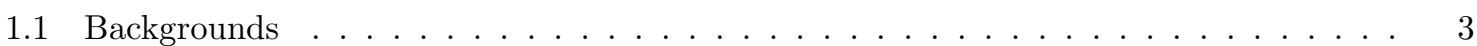

1.2 Branching random walks in random environment (BRWRE) . . . . . . . . . . . . 4

1.3 The associated directed polymers in random environment . . . . . . . . . . . . 4

2 The results 5

2.1 Criteria for global and local survival, and growth rates . . . . . . . . 5

2.2 More on the survival probability . . . . . . . . . . . . 8

3 Preliminaries 9

3.1 Construction of BRWRE . . . . . . . . . . . . . . . . . . . 9

3.2 Complements on DPRE . . . . . . . . . . . . . . . . . . . . . 11

4 Proofs $\quad \mathbf{1 2}$

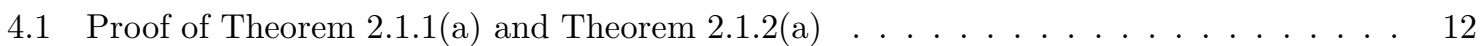

4.2 Local survival and growth rate along subsequences of times . . . . . . . . . . . . 13

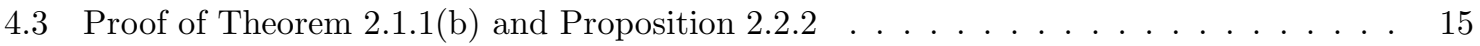

4.4 Local survival and growth rate at all large enough times . . . . . . . . . . . . 17

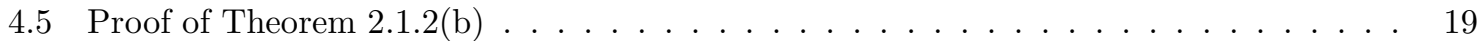

4.6 Generating function of BRWRE . . . . . . . . . . . . . . . . 20

5 Appendix $\mathbf{2 3}$

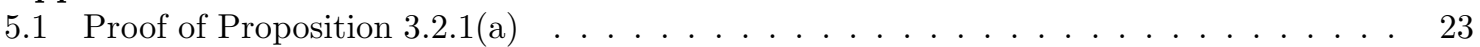

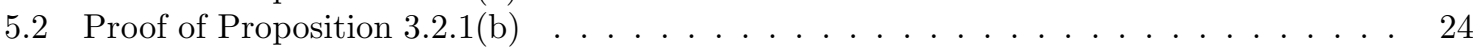

\footnotetext{
${ }^{1}$ March 4, 2019

${ }^{2}$ Partially supported by ANR Polintbio

${ }^{3}$ Partially supported by JSPS Grant-in-Aid for Scientific Research, Kiban (C) 21540125
} 


\section{Introduction}

We consider particles in $\mathbb{Z}^{d}$, performing random walks and branching into independent copies at each step. There is initally one particle located in the origin. When a particle occupies a site $x \in \mathbb{Z}^{d}$ at time $t \in \mathbb{N}=\{0,1, \ldots\}$, then, it moves to a randomly choosen adjacent site $y$ at time $t+1$ and is replaced by $k$ new particles with probability $q_{t, x}(k)$ $(k \in \mathbb{N})$. We assume that the offspring distributions $q_{t, x}=\left(q_{t, x}(k)\right)_{k \in \mathbb{N}}$ form an i.i.d. sequence indexed by time $t$ and space $x$ variables, and we refer to this sequence as the environment. The above model, that we call branching random walks in (space-time) random environment and abreviate as BRWRE, was introduced by Birkner in his thesis [5] under the supervised by Wakolbinger, to analyse interactions between particles sharing the same environment, but independent otherwise. Birkner, Geiger and Kersting [6] obtain sufficient conditions for survival and for extinction, $\mathrm{Hu}$ and Yoshida investigate the localization properties of the empirical particle density in [15], while the second author studies in [25] the diffusive behaviour for $d \geq 3$ and when the diffusion dominates the disorder of the environment. See also an improvement of [25] by Nakashima [19].

Let $B_{t}$ be the set of particles in BRWRE at time $t$, and denote the survival event by

$$
\left\{\text { survival } \stackrel{\text { def. }}{=}\left\{B_{t} \neq \emptyset \text { for all } t \geq 0\right\}\right.
$$

The first fundamental question we address in this paper, is:

When is the probability of the above event (survival probability) positive?

When it is positive indeed, a further natural question will be:

How fast does the total population $\left|B_{t}\right|$ grow as $t \rightarrow \infty$ ?

As will be explained in section 1.3 below, the BRWRE is naturally associated with a model of directed polymers in random environment (DPRE) - see e.g. [7, 9, 10] -, which describes the population mean in a fixed environment. We will show that questions (1.2)-(1.3) intrinsically relate to the behavior of the associated DPRE, and we will answer them in terms of the free energy for the DPRE. We will prove that survival occurs with positive probability when the free energy is positive, while extinction occurs almost surely when it is negative. On the event of survival, the growth rate for the population is the same as for its expectation given the environment. The latter one can be strictly smaller than the one for the unconditional expectation. Besides the overall population, we will consider also the set of particles moving in a specific direction. An interesting point is that martingale theory does not work well in this problem for a large range of parameters (offspring distribution, environmental law, space dimension, ....), neither under the annealed law nor the quenched one, and we will have to resort to different techniques.

Other models of branching random walks in random environment are often considered in the literature. A first one, introduced in [22], is when the offspring distribution is a random i.i.d. sequence depending on time only; we will call it the Smith-Wilkinson model, and it plays a crucial role for comparison with ours. Another popular model is when the offspring distribution is a random i.i.d. sequence depending on space only; our model relates to that one in space dimension $d+1$ by adding an (infinitely) large drift in the first direction, or, equivalently, by considering crossings in that direction. In that model, the interest for global/local growth rates started in [13] in one spatial dimension, allowing explicit results, and in [8] for higher dimension but in the absence of extinction. We emphasize that the possibility of extinction makes the study of growth rates much more delicate. 
Notations We write $\mathbb{N}^{*}=\{1,2, \ldots\}=\mathbb{N} \backslash\{0\}$. For $x \in \mathbb{R}^{d},|x|$ denotes the $\ell^{1}$ norm: $|x|=\left|x_{1}\right|+\ldots+\left|x_{d}\right|$. We write $P[X]=\int X d P$ and $P[X: A]=\int_{A} X d P$ for a random variable $X$ and an event $A$ on some probability space. For two events $A, B$, we write $A \subset B$ a.s. iff $P(A \backslash B)=0$. Similarly, $A=B$ a.s. means that $P(A \backslash B)=P(B \backslash A)=0$. We denote by $\mathcal{P}(\mathbb{N})=\left\{\mathbf{q}=(q(k))_{k \in \mathbb{N}} \in[0,1]^{\mathbb{N}}: \sum_{k \in \mathbb{N}} q(k)=1\right\}$ the set of probability measures on $\mathbb{N}$.

\section{$1.1 \quad$ Backgrounds}

We begin by recalling some classical results for the Galton-Watson process and the SmithWilkinson process. To make the notation consistent with later ones, we formulate these processes as the time evolutions of the set $B_{t}$ of particles, rather than the number of particles. We shortly describe the evolution, leaving a more formal definition for subsequent sections. Let $\mathbf{q}=\left(q_{t}\right)_{t \in \mathbb{N}}$ be a sequence of probability measures on $\mathbb{N}$ such that

$$
q_{t}(1)<1 \text { and } m_{t} \stackrel{\text { def. }}{=} \sum_{k \geq 1} k q_{t}(k)<\infty, \quad \text { for all } t \in \mathbb{N} \text {. }
$$

Then, the branching process with offspring distribution $\mathbf{q}=\left(q_{t}\right)_{t \in \mathbb{N}}$, whose law is denoted by $P^{\mathbf{q}}$, is described as the following dynamics:

- At time $t=0$, there is one particle $\left(\left|B_{0}\right|=1\right)$.

- Each particle in $B_{t}$ will die at time $t+1$, it gives birth to a random number of children with law $q_{t}$ and all these variables are independent.

The Galton-Watson process can be thought of as the simplest process defined as above, where the offspring distribution is time-independent, $q_{t} \equiv q$ for all $t \in \mathbb{N}$. We then write $P=P^{\mathbf{q}}$ in short. As is well known, the answer to the questions (1.2)-(1.3) for the Galton-Watson process is given by:

Theorem 1.1.1 a) [3] $P($ survival $)>0$ if and only if $m \stackrel{\text { def }}{=} \sum_{k \geq 1} k q(k)>1$.

b) [3, 16] Suppose that $m>1$ and $\sum_{k \geq 1} q(k) k \ln k<\infty$. Then

$$
\{\text { survival }\}=\left\{\lim _{t \rightarrow \infty}\left|B_{t}\right| / m^{t} \in(0, \infty)\right\}, \quad P \text {-a.s. }
$$

On the other hand, the Smith-Wilkinson process is defined as above, with $\mathbf{q}=\left(q_{t}\right)_{t \in \mathbb{N}}$ a sequence of i.i.d. random probability measures on $\mathbb{N}$. We denote the law of $\mathbf{q}$ by $Q$ and set

$$
P(\cdot)=\int Q(d \mathbf{q}) P^{\mathbf{q}}(\cdot) .
$$

The answer to the questions (1.2)-(1.3) for the Smith-Wilkinson process was given by W. L. Smith and W. Wilkinson [23], and by K. B. Athreya and S. Karlin [1, 2.

Theorem 1.1.2 Suppose for simplicity that $Q\left[\left|\ln m_{t}\right|\right]<\infty$ (cf. 1.4)). Then,

a) [23, Theorem 3.1], [1, Theorems 1-3]. Either

$$
P^{\mathbf{q}}(\text { survival })>0, Q \text {-a.s. } \text { or } \quad P^{\mathbf{q}}(\text { survival })=0, Q \text {-a.s. }
$$

The former case holds if and only if

$$
Q\left[\ln m_{t}\right]>0 \text { and } Q \ln \frac{1}{1-q_{t}(0)}<\infty .
$$


b) [2, Theorem 1]. In addition to (1.5), suppose that

$$
Q\left[m_{t}^{-1} \sum_{k \geq 1} q_{t}(k) k \ln k\right]<\infty .
$$

Then,

$$
\{\text { survival }\}=\left\{\lim _{t \rightarrow \infty} \frac{\left|B_{t}\right|}{m_{0} \cdots m_{t-1}} \in(0, \infty)\right\}, \quad P \text {-a.s. }
$$

\subsection{Branching random walks in random environment (BRWRE)}

We now introduce the model of interest. To each $(t, x) \in \mathbb{N} \times \mathbb{Z}^{d}$, we associate a distribution $q_{t, x}=\left(q_{t, x}(k)\right)_{k \in \mathbb{N}} \in \mathcal{P}(\mathbb{N})$ on the integers, which serves as an environment. Given $\mathbf{q}=$ $\left(q_{t, x} ;(t, x) \in \mathbb{N} \times \mathbb{Z}^{d}\right)$, we define the branching random walk $(\mathrm{BRW})$, and we denote by $P^{\mathbf{q}}$ its law, as the following dynamics:

- At time $t=0$, there is one particle at the origin $x=0$.

- Each particle, located at site $x \in \mathbb{Z}^{d}$ at time $t$, jumps at time $t+1$ to one of the $2 d$ neighbors of $x$ chosen uniformly; upon arrival, it dies, leaving $k$ new particles there with probability $q_{t, x}(k)$. The number of newborn particles is independent of the jump, and all these variables, indexed by the full population at time $t$, are independent.

In the model of BRW with space-time random environment, we assume that $\mathbf{q}=\left(q_{t, x} ;(t, x) \in\right.$ $\left.\mathbb{N} \times \mathbb{Z}^{d}\right)$ is an i.i.d. sequence in $\mathcal{P}(\mathbb{N})$ with some distribution $Q$. We set $P=\int Q(d \mathbf{q}) P^{\mathbf{q}}$ as before.

As is already mentioned, we denote by $B_{t}$ the set of particles present in the population at time $t$, and $B_{t, x}$ the set of those which are at site $x$ at time $t$. Though this model is the same as in [15, 25], we will formulate it in a detailed manner, by representing each particle by its genealogy. This will provide some nice monotonicity properties, like (4.6).

\subsection{The associated directed polymers in random environment}

From here on, we assume that

$$
Q\left[m_{0,0}+m_{0,0}^{-1}\right]<\infty, \text { where } m_{t, x}=\sum_{k \in \mathbb{N}} k q_{t, x}(k), \quad(t, x) \in \mathbb{Z}^{d} .
$$

We write

$$
m \stackrel{\text { def. }}{=} Q\left[m_{0,0}\right]<\infty .
$$

Let $\left(S_{t}\right)_{t \in \mathbb{N}}$ be the symmetric simple random walk on $\mathbb{Z}^{d}$ starting from $S_{0} \equiv 0$ (cf. (3.3)). We assume that $\left(S_{t}\right)_{t \in \mathbb{N}}$ is defined on a probability space $\left(\Omega_{S}, \mathcal{F}_{S}, P_{S}\right)$. We then introduce the partition functions of directed polymers in random environment (DPRE),

$$
Z_{t, x}=P_{S}\left[\zeta_{t}: S_{t}=x\right] \text { and } Z_{t}=P_{S}\left[\zeta_{t}\right],
$$

where

$$
\zeta_{t}=\prod_{u=0}^{t-1} m_{u, S_{u}}
$$

with $m_{t, x}$ from (1.7). It is easy to see (e.g., [25, Lemma 1.3.1]) that

$$
Z_{t, x}=P^{\mathbf{q}}\left[\left|B_{t, x}\right|\right] \text { and } Z_{t}=P^{\mathbf{q}}\left[\left|B_{t}\right|\right]
$$


For more information on DPRE, see [7, 9, 10] for example. In standard literature on DPRE, the product in (1.10) is defined as $\prod_{u=1}^{t}$ rather than $\prod_{u=0}^{t-1}$. This does not change large $t$ asymptotics, and the results we quote from there in the sequel are not affected by this slight difference.

We set

$$
\Delta=\left\{\theta \in \mathbb{R}^{d} ;|\theta| \leq 1\right\}
$$

and, for $\theta \in \Delta \cap \mathbb{Q}^{d}$,

$$
\mathbb{N}^{*}(\theta)=\left\{t \in \mathbb{N}^{*} ; t \theta \in \mathbb{Z}^{d}, t-t|\theta| \in 2 \mathbb{N}\right\}, \quad \mathbb{N}(\theta)=\{0\} \cup \mathbb{N}^{*}(\theta) .
$$

We have $P_{S}\left(S_{t}=t \theta\right) \geq(2 d)^{-t}>0$ for all $t \in \mathbb{N}(\theta)$. Note also that,

$$
\mathbb{N}(\theta)=n(\theta) \mathbb{N}, \quad \text { with } n(\theta)=\min \mathbb{N}^{*}(\theta) .
$$

Observe that (1.7), combined with the elementary inequality $|\ln u| \leq u \vee u^{-1}$ for $u>0$, implies that

$$
\begin{array}{ll}
Q\left|\ln Z_{t}\right|<\infty & \text { for all } t \geq 1, \\
Q\left|\ln Z_{t, x}\right|<\infty & \text { for all }(t, x) \in \mathbb{N}^{*} \times \mathbb{Z}^{d} \text { with } P_{S}\left(S_{t}=x\right)>0 .
\end{array}
$$

Proposition 1.3.1 There exists a concave, upper semi-continuous function $\psi: \Delta \rightarrow \mathbb{R}$, with $\Delta$ from (1.12), such that

$$
\psi(\theta)=\lim _{\substack{t \rightarrow \infty \\ t \in \mathbb{N}^{*}(\theta)}} \frac{1}{t} Q\left[\ln Z_{t, t \theta}\right] \text { for all } \theta \in \Delta \cap \mathbb{Q}^{d} .
$$

$\psi$ is symmetric in the sense that $\psi(\theta)=\psi\left(\left|\theta_{\sigma(1)}\right|, \ldots,\left|\theta_{\sigma(d)}\right|\right)$ for any permutation $\sigma$ of $\{1, \ldots, d\}$, and

$$
Q\left[\ln m_{0,0}\right]-\ln (2 d)=\psi\left( \pm e_{i}\right) \leq \psi(\theta) \leq \psi(0) .
$$

Moreover, the following limit also exists and equals $\psi(0)$ :

$$
\Psi \stackrel{\text { def. }}{=} \lim _{t \rightarrow \infty} \frac{1}{t} Q\left[\ln Z_{t}\right]
$$

We refer the reader to [7, page 287, Theorems 1.1 and 1.2] for the proof of Proposition 1.3.1 (Although the random variable $\ln m_{0,0}$ is assumed to be Gaussian in [7, it is not essential for this purpose). The number $\Psi$ is called the free energy of the polymer, and $\psi(\theta)$ the directional free energy.

\section{The results}

\subsection{Criteria for global and local survival, and growth rates}

We suppose (1.7). Then, the growth rate for the total population of the BRWRE is identified with the free energy (1.18) of the DPRE associated to it by (1.9).

Theorem 2.1.1 (global growth) Let $\varepsilon>0$. 
a) We have, P-a.s.,

$$
\left|B_{t}\right| \leq e^{(\Psi+\varepsilon) t} \text { for all large } t \text { 's. }
$$

More precisely, there exist $c_{1}, c_{2} \in(0, \infty)$ such that

$$
P\left(\left|B_{t}\right| \geq e^{(\Psi+\varepsilon) t}\right) \leq 3 \exp \left(-c_{1} \varepsilon^{2} t\right) \quad \text { for all } \varepsilon \in\left(0, c_{2}\right], t \geq 1 .
$$

In particular,

$$
P(\text { survival })=0 \text { if } \Psi<0, \quad c f . \text { (1.1). }
$$

b) Suppose $\Psi>0$ and

$$
Q \ln \frac{1}{1-q_{0,0}(0)}<\infty
$$

Then,

$$
P^{\mathbf{q}}(\text { survival })>0, \quad \text { Q-a.s. }
$$

Suppose $\Psi>0$, (2.3) and

$$
Q\left[\frac{P^{\mathbf{q}}\left[\left|B_{t, 0}\right| \ln \left|B_{t, 0}\right|\right]}{Z_{t, 0}}\right]<\infty \text { for all } t \in 2 \mathbb{N}^{*} .
$$

Then,

$$
\{\text { survival }\}=\left\{\left|B_{t}\right| \geq e^{(\Psi-\varepsilon) t} \text { for all large } t^{\prime} s\right\}, \quad P \text {-a.s. }
$$

In particular, (2.6), together with (2.1) says that $\Psi>0$ implies

$$
\{\text { survival }\}=\left\{\lim _{t \rightarrow \infty} \frac{1}{t} \ln \left|B_{t}\right|=\Psi\right\}, \quad P \text {-a.s. }
$$

under the conditions (2.3) and (2.5). The assumption " $\Psi>0$ and (2.3)" for Theorem 2.1.1(b) is the generalization of (1.5) (also of " $m>1$ " in Theorem 1.1.1), while the condition (2.5) is the counterpart of (1.6). We will explain in the remarks (1)-(2) below that the assumptions in Theorem 2.1.1(b) are not too restrictive, while, in remark (3), we compare the survival probability $P$ (survival) with those of the Galton-Watson process and the Smith-Wilkinson process, which are properly associated with our BRWRE.

Remarks: 1) Assume that $m_{0,0}$ is not a constant a.s. Then, by Proposition 3.2.1 (b), $Q\left[\ln m_{0,0}\right] \geq 0$ (for which $m_{0,0} \geq 1$ a.s. is more than enough) implies that $\Psi>0$.

2) We will see at the end of section 2.1 that the following mild integrability condition implies both (2.3) and (2.5).

$$
Q\left[\ln m_{0,0}^{(2)}\right]<\infty, \text { where } m_{t, x}^{(2)}=\sum_{k \in \mathbb{N}} k^{2} q_{t, x}(k)
$$

3) Let us suppose (2.3) and let $\sigma^{\mathrm{GW}}$ and $\sigma^{\mathrm{SW}}$ be survival probabilities for Galton-Watson model with offspring distribution $Q\left[q_{0,0}(\cdot)\right]$ and the Smith-Wilkinson process with the offspring distributions $q_{t, 0}, t \in \mathbb{N}^{*}$. By computations of generating function in section 4.6 below, we will see that

$$
\sigma^{\mathrm{SW}} \leq P(\text { survival }) \leq \sigma^{\mathrm{GW}} .
$$

On the other hand, it is known that $\sigma^{\mathrm{SW}}>0$ if and only if $Q\left[\ln m_{0,0}\right]>0$ [1, 23]. Thus, by Theorem 2.1.1, we have

$$
\sigma^{\mathrm{SW}}=0<P(\text { survival }) \text { if } Q\left[\ln m_{0,0}\right] \leq 0<\Psi .
$$


Similarly, we have

$$
P(\text { survival })=0<\sigma^{\mathrm{GW}} \text { if } \Psi<0<\ln m .
$$

We now turn to the criterion for the local survival and the identification of the local growth rate in terms of the directional growth rate (1.16):

Theorem 2.1.2 (local growth) Let $\theta \in \Delta \cap \mathbb{Q}^{d}$ and $\varepsilon>0$. Then,

a) P-almost surely,

$$
\left|B_{t, t \theta}\right| \leq e^{(\psi(\theta)+\varepsilon) t} \text { for all large } t \in \mathbb{N} .
$$

More precisely, there exist $c_{1}, c_{2} \in(0, \infty)$ such that

$$
P\left(\left|B_{t, t \theta}\right| \geq e^{(\psi(\theta)+\varepsilon) t}\right) \leq 3 \exp \left(-c_{1} \varepsilon^{2} t\right)
$$

for all $\varepsilon \in\left(0, c_{2}\right]$ and $t \in \mathbb{N}$. In particular,

$$
P\left(B_{t, t \theta} \neq \emptyset \text { i.o. }\right)=0 \text { if } \psi(\theta)<0,
$$

b) Supppose $\psi(\theta)>0$ and (2.3). Then,

$$
P^{\mathbf{q}}\left(B_{t, t \theta} \neq \emptyset \text { for all } t \in \mathbb{N}(\theta)\right)>0, \quad Q \text {-a.s. }
$$

Supppose $\psi(\theta)>0$, (2.3) and

$$
Q\left[\frac{P^{\mathbf{q}}\left[\left|B_{t, t \theta}\right| \ln \left|B_{t, t \theta}\right|\right]}{Z_{t, t \theta}}\right]<\infty \text { for all } t \in \mathbb{N}^{*}(\theta) .
$$

Then,

$$
\left\{B_{t, t \theta} \neq \emptyset \text { for all } t \in \mathbb{N}(\theta)\right\} \subset\left\{\left|B_{t, t \theta}\right| \geq e^{(\psi(\theta)-\varepsilon) t} \text { for all large } t \in \mathbb{N}(\theta)\right\}, \quad \text { P-a.s. }
$$

Remarks: 1) Since $\psi: \Delta \rightarrow \mathbb{R}$ is concave, it is continuous in the interior of $\Delta$ [21, page 82, Theorem 10.1]. Hence, if $\Psi=\psi(0)>0$, then $\psi(\theta)>0$ in a neighborhood of 0 .

2) We will see at the end of section 2.1 that the mild integrability condition (2.8) implies both (2.3) and (2.13).

We close this subsection with the proof that (2.8) implies (2.3) and (2.13) for all $\theta \in \Delta \cap \mathbb{Q}^{d}$. To show (2.3), we note that $1-q_{0,0}(0)=\sum_{k \geq 1} q_{0,0}(k)$ and hence that

$$
m_{0,0}^{2}=\left(\sum_{k \geq 1} k q_{0,0}(k)\right)^{2} \stackrel{\text { Schwarz }}{\leq}\left(1-q_{0,0}(0)\right) m_{0,0}^{(2)} .
$$

Therefore,

$$
\ln \frac{1}{1-q_{0,0}(0)} \leq \ln \frac{m_{0,0}^{(2)}}{m_{0,0}^{2}}=\ln m_{0,0}^{(2)}-2 \ln m_{0,0},
$$

which proves that (2.8) implies (2.3) .

To show (2.13), we note that

$$
\frac{P^{\mathbf{q}}\left[\left|B_{t, t \theta}\right| \ln \left|B_{t, t \theta}\right|\right]}{Z_{t, t \theta}} \stackrel{\text { Jensen }}{\leq} \ln \frac{P^{\mathbf{q}}\left[\left|B_{t, t \theta}\right|^{2}\right]}{Z_{t, t \theta}}=\ln P^{\mathbf{q}}\left[\left|B_{t, t \theta}\right|^{2}\right]-\ln Z_{t, t \theta}
$$

Thus, in view of (1.15), it is enough to prove that 
To this end, we write

$$
\left|B_{t}\right|^{2}=\left(\sum_{(x, \nu) \in B_{t-1}} K_{t-1, x, \nu}\right)^{2} \leq\left|B_{t-1}\right| \sum_{(x, \nu) \in B_{t-1}} K_{t-1, x, \nu}^{2}
$$

and

$$
P^{\mathbf{q}}\left[\left|B_{t}\right|^{2} \mid \mathcal{F}_{t-1}\right] \leq\left|B_{t-1}\right| \sum_{(x, \nu) \in B_{t-1}} m_{t-1, x}^{(2)} \leq\left|B_{t-1}\right|^{2} \max _{|x| \leq t-1} m_{t-1, x}^{(2)} .
$$

By iteration, we get

$$
P^{\mathbf{q}}\left[\left|B_{t}\right|^{2}\right] \leq \prod_{u=0}^{t-1} \max _{|x| \leq u} m_{u, x}^{(2)}
$$

and hence

$$
\ln P^{\mathbf{q}}\left[\left|B_{t}\right|^{2}\right] \leq \sum_{u=0}^{t-1} \max _{|x| \leq u} \ln m_{u, x}^{(2)} \leq \sum_{u=0}^{t-1} \sum_{|x| \leq u} \ln m_{u, x}^{(2)},
$$

which proves that (2.8) implies (1).

\subsection{More on the survival probability}

There are still additional observations which we can make on the survival probability. The first of these is the following zero-one law:

\section{Proposition 2.2.1 Either}

$$
P^{\mathbf{q}}(\text { survival })>0 \quad \text { Q-a.s. } \text { or } \quad P^{\mathbf{q}}(\text { survival })=0 \quad \text { Q-a.s. }
$$

The proof of Proposition 2.2.1 is presented in section 4.6. We already know the zero-one law when $\Psi \neq 0$ from Theorem 2.1.1, but the result is new in the critical case $\Psi=0$. Since $\left|B_{t}\right|$ is an integer, quantization effects supports the following conjecture, that we leave for future work.

Conjecture 2.2.1 When $\Psi=0, P$ (survival) $=0$.

Another observation is concerned with the non-degeneracy of the limit of a martingale, which is obtained by normalizing the total population $\left|B_{t}\right|$. To state it, we set

$$
W_{t}=\left|B_{t}\right| / m^{t},
$$

where $m$ is defined by (1.8). The non-negative sequence $W_{t}$ is a $\left(P,\left(\mathcal{F}_{t}\right)\right)$-martingale, and by Doob's martingale convergence theorem [12], the following limit exists:

$$
W_{\infty}=\lim _{t} W_{t}, \quad P \text {-a.s. }
$$

It is known [25, Corollaries 1.2.2 and 3.3.2] that

$$
P\left(W_{\infty}>0\right) \begin{cases}>0 & \text { if }(3.18) \\ =0 & \text { if (3.19). }\end{cases}
$$

In particular, there is a whole range of parameters where $P\left(W_{\infty}>0\right)=0<P$ (survival), namely, when both (3.19) and $\Psi>0$ hold. However, arguments used to prove Theorem 2.1.1 (Lemma 4.3.1 below) also lead to: 
Proposition 2.2.2 Suppose that $m<\infty$ and $P\left(W_{\infty}>0\right)>0$. Then,

$$
\{\text { survival }\} \stackrel{\text { a.s. }}{=}\left\{W_{\infty}>0\right\} \stackrel{\text { a.s. }}{=}\left\{\lim _{t \rightarrow \infty} \frac{\left|B_{t}\right|}{Z_{t}} \in(0, \infty)\right\} \text {. }
$$

The above proposition is an analogue of a classical result for the Galton-Watson process [3. page 9, Theorem 9 (iii)] (Note that the hypothesis $\sigma^{2}<\infty$ is not used there, but only $m<\infty)$. It extends to BRWRE, Theorem 1.1.1 (b) and Theorem 1.1.2 (b). Under an additional assumption, it improves (2.7). We also remark that the equality analogous to Proposition 2.2.2 holds true for continuous and discrete time linear systems [14, 26] taking values in $\mathbb{N}^{d}$. Finally, note that non-degenerate limits for local ratios $\left|B_{t, x}\right| / Z_{t, x}$ have been found for homogeneous BRW [4], but in the case of random environment this seems to be beyond reach.

\section{Preliminaries}

\subsection{Construction of BRWRE}

To give a precise definition of the above dynamics, we will adopt here a reformulation given in [19], and introduce a tree which consists of all possible ancestral histories of the branching process:

$$
\mathcal{T}=\bigcup_{t \in \mathbb{N}} \mathcal{T}_{t} \quad \text { where } \mathcal{T}_{t}=\left\{\nu=\left(\nu_{i}\right)_{i=0}^{t} \in\left(\mathbb{N}^{*}\right)^{1+t} ; \nu_{0}=1\right\}
$$

For $\nu \in \mathcal{T},|\nu| \in \mathbb{N}$ stands for its generation defined by $\nu \in \mathcal{T}_{|\nu|}$. For $\nu \in \mathcal{T}_{t+s},\left.\nu\right|_{t}$ denotes the ancestral history of $\nu$ up to generation $t$ :

$$
\left.\nu\right|_{t}=\left(1, \nu_{1}, \ldots, \nu_{t}\right) \in \mathcal{T}_{t} .
$$

Let $p(\cdot, \cdot)$ be a transition probability for the symmetric simple random walk on $\mathbb{Z}^{d}$ :

$$
p(x, y)= \begin{cases}\frac{1}{2 d} & \text { if }|x-y|=1 \\ 0 & \text { if }|x-y| \neq 1\end{cases}
$$

Fix an environment $\mathbf{q}=\left(q_{t, x}\right)_{(t, x) \in \mathbb{N} \times \mathbb{Z}^{d}}, q_{t, x} \in \mathcal{P}(\mathbb{N})$. Then, the branching random walk (BRW) with offspring distribution $\mathbf{q}$ is described in an unformal manner as follows:

- At time $t=0$, there is one particle at the origin $x=0$ (the founding ancestor labeled by "1").

- Suppose that there is a particle at site $x \in \mathbb{Z}^{d}$ at time $t$, which is labeled by $\nu=$ $\left(1, \nu_{1}, . ., \nu_{t}\right), \nu_{i} \in \mathbb{N}^{*}$. At time $t+1$, it jumps to a site $y$ with probability $p(x, y)$ independently of the other particles. At arrival, it dies, leaving $k$ new particles there with probability $q_{t, x}(k)$. The newborn particles are labeled by $\left(1, \nu_{1}, \ldots, \nu_{t}, \nu_{t+1}\right)$ (with $\left.\nu_{t+1}=1, \ldots, k\right)$.

To put it on steady grounds, we introduce a few ingredients.

- Spatial motion: We define the measurable space $\left(\Omega_{X}, \mathcal{F}_{X}\right)$ as the set $\left(\mathbb{Z}^{d}\right)^{\mathbb{N} \times \mathbb{Z}^{d} \times \mathcal{T}}$ with the product $\sigma$-field, and $\Omega_{X} \ni X \mapsto X_{t, x, \nu}$ for each $(t, x, \nu) \in \mathbb{N} \times \mathbb{Z}^{d} \times \mathcal{T}$ as the projection. We define $P_{X} \in \mathcal{P}\left(\Omega_{X}, \mathcal{F}_{X}\right)$ as the product measure such that

$$
P_{X}\left(X_{t, x, \nu}=y\right)=p(x, y) \text { for all }(t, x, \nu) \in \mathbb{N} \times \mathbb{Z}^{d} \times \mathcal{T} \text { and } y \in \mathbb{Z}^{d} .
$$


Here, we interpret $X_{t, x, \nu}$ as the position at time $t+1$ of the children born from the particle labeled by $\nu$, when it occupies the time-space location $(t, x)$.

- Offspring distribution: We set $\Omega_{q}=\mathcal{P}(\mathbb{N})^{\mathbb{N} \times \mathbb{Z}^{d}}$. Thus, each $\mathbf{q} \in \Omega_{\mathbf{q}}$ is a function $(t, x) \mapsto$ $q_{t, x}=\left(q_{t, x}(k)\right)_{k \in \mathbb{N}}$ from $\mathbb{N} \times \mathbb{Z}^{d}$ to $\mathcal{P}(\mathbb{N})$. We interpret $q_{t, x}$ as the offspring distribution for each particle which occupies the time-space location $(t, x)$. The set $\mathcal{P}(\mathbb{N})$ is equipped with the natural Borel $\sigma$-field induced from that of $[0,1]^{\mathbb{N}}$. We denote by $\mathcal{F}_{\mathbf{q}}$ the product $\sigma$-field on $\Omega_{\mathbf{q}}$.

We define the measurable space $\left(\Omega_{K}, \mathcal{F}_{K}\right)$ as the set $\mathbb{N}^{\mathbb{N} \times \mathbb{Z}^{d} \times \mathcal{T}}$ with the product $\sigma$-field, and $\Omega_{K} \ni K \mapsto K_{t, x, \nu}$ for each $(t, x, \nu) \in \mathbb{N} \times \mathbb{Z}^{d} \times \mathcal{T}$ as the projection. For each fixed $\mathbf{q} \in \Omega_{\mathbf{q}}$, we define $P_{K}^{\mathbf{q}} \in \mathcal{P}\left(\Omega_{K}, \mathcal{F}_{K}\right)$ as the product measure such that

$$
P_{K}^{\mathbf{q}}\left(K_{t, x, \nu}=k\right)=q_{t, x}(k) \text { for all }(x, t, \nu) \in \mathbb{N} \times \mathbb{Z}^{d} \times \mathcal{T} \text { and } k \in \mathbb{N} .
$$

We interpret $K_{t, x, \nu}$ as the number of the children born from the particle $\nu$ at time-space location $(t, x)$.

- Branching random walk in random environment (BRWRE): We fix a product measure $Q \in \mathcal{P}\left(\Omega_{\mathbf{q}}, \mathcal{F}_{\mathbf{q}}\right)$, which describes the i.i.d. offspring distribution assigned to each time-space location. Finally, we define $(\Omega, \mathcal{F})$ by

$$
\Omega=\Omega_{X} \times \Omega_{K} \times \Omega_{\mathbf{q}}, \quad \mathcal{F}=\mathcal{F}_{X} \otimes \mathcal{F}_{K} \otimes \mathcal{F}_{\mathbf{q}},
$$

and $P^{\mathbf{q}}, P \in \mathcal{P}(\Omega, \mathcal{F})$ by

$$
P^{\mathbf{q}}=P_{X} \otimes P_{K}^{\mathbf{q}} \otimes \delta_{\mathbf{q}}, \quad P=\int Q(d \mathbf{q}) P^{\mathbf{q}}
$$

We define a Markov chain $\left(B_{t}\right)_{t \in \mathbb{N}}$ with values in finite subsets of $\mathbb{Z}^{d} \times \mathcal{T}$, inductively by $B_{0}=(0,1)$, and, for $t \geq 1$,

$$
B_{t}=\bigcup_{(x, \nu) \in B_{t-1}}\left\{(y, \mu) \in \mathbb{Z}^{d} \times \mathcal{T}_{t} ; X_{t-1, x, \nu}=y,\left.\mu\right|_{t-1}=\nu, \mu_{t} \leq K_{t-1, x, \nu}\right\} .
$$

We call the above process the branching random walk in random environment (BRWRE). It is meant by $(x, \nu) \in B_{t}$ that the time-space location $(t, x)$ is occupied by a particle with its ancestoral history $\nu$. We consider the filtration:

$$
\mathcal{F}_{0}=\{\emptyset, \Omega\}, \quad \mathcal{F}_{t}=\sigma\left(X_{s, \cdot,}, K_{s, \cdot,}, q_{s, .} ; s \leq t-1\right), \quad t \geq 1,
$$

which the process $\left(B_{t}\right)_{t \in \mathbb{N}}$ is adapted to. We define, for fixed $(t, x) \in \mathbb{N} \times \mathbb{Z}^{d}$, the set of particles in $B_{t}$, which occupies the site time-space $(t, x)$ is denoted by:

$$
B_{t, x}=\left\{(y, \nu) \in B_{t} ; y=x\right\} .
$$

We remark that the total population $\left|B_{t}\right|$ is exactly the classical Galton-Watson process if $q_{t, x} \equiv q$, where $q \in \mathcal{P}(\mathbb{N})$ is non-random. On the other hand, if $\mathbb{Z}^{d}$ is replaced a singleton, then $\left|B_{t}\right|$ is the population of the Smith-Wilkinson model [23].

Remark: The definition (3.8) is consistent with that in [15, 25]. In fact, it is easy to see from (3.6) that

$$
\left|B_{0, y}\right|=\delta_{0, y}, \quad\left|B_{t, y}\right|=\sum_{(x, \nu) \in B_{t-1}} \delta_{y}\left(X_{t-1, x, \nu}\right) K_{t-1, x, \nu}, \quad t \geq 1 .
$$


If we write

$$
\{\nu(i)\}_{i=1}^{\left|B_{t-1, x}\right|}=\left\{\nu \in \mathcal{T}_{t-1}:(x, \nu) \in B_{t-1}\right\}
$$

for each $x \in \mathbb{Z}^{d}$, then, (3.9) becomes:

$$
\left|B_{0, y}\right|=\delta_{0, y}, \quad\left|B_{t, y}\right|=\sum_{x \in \mathbb{Z}^{d}} \sum_{i=1}^{\left|B_{t-1, x}\right|} \delta_{y}\left(X_{t-1, x, \nu(i)}\right) K_{t-1, x, \nu(i)}, \quad t \geq 1
$$

which is the recursion used in [15, 25].

\subsection{Complements on DPRE}

We denote by $I_{S}$ the large deviation rate function for the random variables $\left(S_{t} / t\right)_{t \in \mathbb{N}^{*}}$ :

$$
I_{S}(\theta)=\sup _{\alpha \in \mathbb{R}^{d}}\left\{\alpha \cdot \theta-\ln P_{S}\left[\exp \left(\alpha \cdot S_{1}\right)\right]\right\}, \quad \theta \in \Delta .
$$

It is well-known that

$$
0=I_{S}(0) \leq I_{S}(\theta) \leq I_{S}\left( \pm e_{i}\right)=\ln (2 d),
$$

where $e_{i}=\left(\delta_{i, j}\right)_{j=1}^{d}$, and, for all $\theta \in \Delta \cap \mathbb{Q}^{d}$, that

$$
-I_{S}(\theta)=\lim _{\substack{t \rightarrow \infty \\ t \in \mathbb{N}^{*}(\theta)}} \frac{1}{t} \ln P_{S}\left(S_{t}=t \theta\right)=\sup _{t \in \mathbb{N}^{*}(\theta)} \frac{1}{t} \ln P_{S}\left(S_{t}=t \theta\right) .
$$

Proposition 3.2.1 a) We have

$$
Q\left[\ln m_{0,0}\right]-I_{S}(\theta) \leq \psi(\theta) \leq \ln m-I_{S}(\theta) \text { for all } \theta \in \Delta \cap \mathbb{Q}^{d} .
$$

(Note that $Q\left[\left|\ln m_{0,0}\right|\right]<\infty$ by [1.7)). Moreover, the first inequality is strict if $\theta=0$ and $m_{0,0} \not \equiv m$ a.s. Finally, the first inequality in (3.14) is an equality if $\theta= \pm e_{i}$.

b) There are constants $c_{1}, c_{2} \in(0, \infty)$ such that

$$
Q\left(\frac{1}{t}\left|\ln Z_{t}-Q\left[\ln Z_{t}\right]\right|>\varepsilon\right) \leq 2 \exp \left(-c_{1} \varepsilon^{2} t\right)
$$

for all $\varepsilon \in\left(0, c_{2}\right]$ and $t \in \mathbb{N}^{*}$, and

$$
Q\left(\frac{1}{t}\left|\ln Z_{t, t \theta}-Q\left[\ln Z_{t, t \theta}\right]\right|>\varepsilon\right) \leq 2 \exp \left(-c_{1} \varepsilon^{2} t\right)
$$

for all $\varepsilon \in\left(0, c_{2}\right], \theta \in \Delta \cap \mathbb{Q}^{d}$ and $t \in \mathbb{N}^{*}(\theta)$. As a consequence,

$$
\lim _{t \rightarrow \infty} \frac{1}{t} \ln Z_{t}=\Psi \quad \text { and } \quad \lim _{\substack{t \rightarrow \infty \\ t \in \mathbb{N}^{*}(\theta)}} \frac{1}{t} \ln Z_{t, t \theta}=\psi(\theta), \quad Q \text {-a.s. }
$$

We prove Proposition 3.2.1(a) in section 5.1. A way to prove Proposition 3.2.1(b) is to apply a series of general martingale inequalities as is done in [18], theorem 7.2. For the reader's convenience, we enclose a short proof in section 5.2 . 
Remarks: 1) By (3.17) (also as is discussed in [7]), the function $\psi(0)-\psi(\cdot): \Delta \rightarrow[0, \infty$ ) gives, for almost all realizations of the environment, the large deviation rate function for the random probability measures (usually called polymer measures):

$$
\frac{1}{Z_{t}} P_{S}\left[\zeta_{t}: S_{t} / t \in \cdot\right] \quad \text { as } t \rightarrow \infty \text {. }
$$

2) The quantity $\Psi$ is called the free energy in the context of DPRE. Its value relative to $\ln m$ is important there, and hence is well studied. $\Psi \leq \ln m$ by Proposition 3.2 .1 (b). $\Psi=\ln m$ if

$$
d \geq 3 \text { and } \frac{Q\left[m_{0,0}^{2}\right]}{m^{2}}<\frac{1}{\pi_{d}},
$$

where $\pi_{d}=P_{S}\left(S_{t}=0\right.$ for some $\left.t \geq 1\right)$. More precisely, $\left(Z_{t} / m^{t}\right)_{t \geq 1}$ converges to a positive limit $Q$-a.s. under the condition (3.18) ([10, page 128, Remark 3.2.3], [24, page 282, Lemma 1]). On the other hand, $\Psi<\ln m$ if

$$
\begin{aligned}
& d=1,2 \text { and } m_{0,0} \text { is not a constant, } \\
& \text { or } Q\left[\frac{m_{0,0}}{m} \ln \frac{m_{0,0}}{m}\right]>\ln (2 d)
\end{aligned}
$$

([9, page 709, Theorem 2.3(a)], [11, 17]).

\section{Proofs}

\subsection{Proof of Theorem 2.1.1(a) and Theorem 2.1.2(a)}

We first show (2.2). By superadditivity (e.g., [9, page 720, Proof of Proposition 2.5]) and Proposition 3.2.1, we have for large $T$ that

$$
Q\left[\ln Z_{T}\right] \geq T \Psi \text { and } Q\left(\ln Z_{T} \geq Q\left[\ln Z_{T}\right]+\frac{\varepsilon}{2} T\right) \leq 2 \exp \left(-c_{1} \varepsilon^{2} T\right) .
$$

Thus,

1) $Q\left(Z_{T} \geq e^{\left(\Psi+\frac{\varepsilon}{2}\right) T}\right) \leq 2 \exp \left(-c_{1} \varepsilon^{2} T\right)$.

On the other hand, we have for for any $\chi=\chi(\mathbf{q}) \in\{0,1\}$ that

$$
\begin{gathered}
P^{\mathbf{q}}\left(\left|B_{T}\right| \geq e^{(\Psi+\varepsilon) T}\right) \leq P^{\mathbf{q}}\left(\left|B_{T}\right| \geq e^{(\Psi+\varepsilon) T}\right) \chi+1-\chi \\
\stackrel{\substack{\text { Chebyshev } \\
\leq}}{\leq} e^{-(\Psi+\varepsilon) T} Z_{T} \chi+1-\chi .
\end{gathered}
$$

We choose $\chi=\mathbf{1}\left\{Z_{T}<e^{\left(\Psi+\frac{\varepsilon}{2}\right) T}\right\}$ and take $Q$-expectation to get

$$
P\left(\left|B_{T}\right| \geq e^{(\Psi+\varepsilon) T}\right) \leq e^{-\frac{\varepsilon}{2} T}+Q\left(Z_{T} \geq e^{\left(\Psi+\frac{\varepsilon}{2}\right) T}\right) \stackrel{(1)}{\leq} e^{-\frac{\varepsilon}{2} T}+2 \exp \left(-c_{1} \varepsilon^{2} T\right) .
$$

We now obtain (2.1) by Borel-Cantelli lemma. This proves Theorem 2.1.1(a). The proof of Theorem 2.1.2 (a) is similar. 


\subsection{Local survival and growth rate along subsequences of times}

The purpose of this subsection is to prove the following two lemmas, which proves (2.4) and will also be our first step for the proof of Theorem 2.1.2(b).

Lemma 4.2.1 Suppose $\theta \in \Delta \cap \mathbb{Q}^{d}$, (2.3) and that there exists $T \in \mathbb{N}^{*}(\theta)$ such that

$$
Q\left[\ln Z_{T, T \theta}\right]>0
$$

Then,

$$
P^{\mathbf{q}}\left(B_{s T, s T \theta} \neq \emptyset \text { for all } s \geq 1\right)>0, \quad \text { Q-a.s. }
$$

Suppose in addition that

$$
Q\left[\frac{P^{\mathbf{q}}\left[\left|B_{T, T \theta}\right| \ln \left|B_{T, T \theta}\right|\right]}{Z_{T, T \theta}}\right]<\infty .
$$

Then, for any $1 \leq r<\exp \left(\frac{1}{T} Q\left[\ln Z_{T, T \theta}\right]\right)$,

$$
P^{\mathbf{q}}\left(\varliminf_{s \rightarrow \infty} r^{-s T}\left|B_{s T, s T \theta}\right|>0\right)>0, \quad \text { Q-a.s. }
$$

Lemma 4.2.2 Suppose $\theta \in \Delta \cap \mathbb{Q}^{d}, \psi(\theta)>0$, and (2.3). Then (4.2) holds for any large enough $T \in \mathbb{N}^{*}(\theta)$. If we suppose (2.13) in addition, then, (4.4) holds for any large enough $T \in \mathbb{N}^{*}(\theta)$ and for any $r<\exp (\psi(\theta))$.

Since $\Psi=\psi(0)$, we get (2.4) from Lemma 4.2.2,

Let us first prove Lemma 4.2.2, assuming Lemma 4.2.1,

Let $1 \leq r<\exp (\psi(\theta))$. Then, by (1.16),

$$
\frac{1}{T} Q\left[\ln Z_{T, T \theta}\right]>\ln r \text { for all large } T \in \mathbb{N}^{*}(\theta),
$$

and the claim follows from Lemma 4.2.1.

To prove Lemma 4.2.1, we introduce some notation. For $(s, z, \lambda) \in \mathbb{N} \times \mathbb{Z}^{d} \times \mathcal{T}_{s}$, we define the $(s, z, \lambda)$-branch

$$
\left(B_{t}^{s, z, \lambda}\right)_{t \in \mathbb{N}}
$$

of $\left(B_{t}\right)_{t \in \mathbb{N}}$ inductively by $B_{0}^{s, z, \lambda}=(z, \lambda)$ and for $t \geq 1$,

$$
B_{t}^{s, z, \lambda}=\bigcup_{(x, \nu) \in B_{t-1}^{s, z, \lambda}}\left\{(y, \mu) \in \mathbb{Z}^{d} \times \mathcal{T}_{s+t} ; X_{s+t-1, x, \nu}=y,\left.\mu\right|_{s+t-1}=\nu, \mu_{s+t} \leq K_{s+t-1, x, \nu}\right\} .
$$

This amounts to restarting a BRWRE from a single particle at time-space $(s, z)$, whose ancestoral history up to time $s$ is given by $\lambda$. Clearly,

$$
\left\{(z, \lambda) \in B_{s}\right\} \subset\left\{B_{t}^{s, z, \lambda} \subset B_{s+t} \text { for all } t \geq 0\right\} .
$$

Note also that $B^{s, z, \lambda}$ is a function of

$$
\left\{\left(X_{s+\cdot, \cdot, \nu}, K_{s+\cdot, \cdot, \nu}\right) ;\left.\nu\right|_{s}=\lambda\right\} .
$$

and therefore, for each fixed $(s, z)$,

$$
\left\{B^{s, z, \lambda}\right\}_{\lambda \in \mathcal{T}_{s}} \text { are i.i.d. under } P^{\mathbf{q}} .
$$


For $(t, x) \in \mathbb{N} \times \mathbb{Z}^{d}$, the set of particles in $B_{t}^{s, z, \lambda}$, which occupy the site $x$ is denoted by:

$$
B_{t, x}^{s, z, \lambda}=\left\{(y, \nu) \in B_{t}^{s, z, \lambda} ; y=x\right\} .
$$

We fix a $T$ such that (4.1) holds. We will then define a Markov chain $\left(B_{s}^{*}\right)_{s \in \mathbb{N}}$ with values in finite subsets of $\mathcal{T}$, which serves as a "lower bound" of $\left(B_{s T, s T \theta}\right)_{s \in \mathbb{N}}$. We now define $B_{0}^{*}=\{1\}$, and for $s \geq 1$,

$$
B_{s}^{*}=\bigcup_{\lambda \in B_{s-1}^{*}} B_{s, \lambda}^{*}, \text { with } B_{s, \lambda}^{*}=\left\{\nu \in \mathcal{T}_{s T} ;(s T \theta, \nu) \in B_{T}^{(s-1) T,(s-1) T \theta, \lambda}\right\} .
$$

In words, $B_{s}^{*}$ is the subset of $B_{s T, s T \theta}$ composed of genealogies which have been at site $r T \theta$ at times $r T$ for $r=1,2, \ldots, s$.

Lemma 4.2.3 a) For each $s \geq 1,\left\{B_{s, \lambda}^{*}\right\}_{\lambda \in \mathcal{T}_{(s-1) T}}$ are i.i.d. under $P^{\mathbf{q}}$.

b) Define $q_{s}^{*} \in \mathcal{P}(\mathbb{N}), s \in \mathbb{N}$ by

$$
q_{s}^{*}(k)=P^{\mathbf{q}}\left(\left|B_{s, \lambda}^{*}\right|=k\right), \quad k \in \mathbb{N} .
$$

Then, the sequence $\left(q_{s}^{*}\right)_{s \in \mathbb{N}}$ is i.i.d. under $P$.

Proof: a) :This follows from (4.7).

b):Since $q_{s}^{*}$ is $\mathcal{F}_{s T}$-measurable, it is enough to show that

$$
P\left(q_{s}^{*} \in \cdot \mid \mathcal{F}_{(s-1) T}\right)=P\left(q_{1}^{*} \in \cdot\right) .
$$

Let $\theta_{t, x}: \omega \mapsto \theta_{t, x} \omega$ be time-space shift for $\omega=(X, K, q)$. Then, $q_{s}^{*}=q_{1}^{*} \circ \theta_{(s-1) T,(s-1) T \theta}$. By the shift-invariance, we have

$$
P\left(q_{s}^{*} \in \cdot \mid \mathcal{F}_{(s-1) T}\right)=P\left(q_{1}^{*} \circ \theta_{(s-1) T,(s-1) T \theta} \in \cdot \mid \mathcal{F}_{(s-1) T}\right)=P\left(q_{1}^{*} \in \cdot\right) .
$$

By Lemma 4.2.3, the sequence $\left(\left|B_{s}^{*}\right|\right)_{s \in \mathbb{N}}$ is a branching process with random environments in the sense of Smith and Wilkinson [23] (See also [1, 2]). We write

$$
m_{s}^{*}=\sum_{k \in \mathbb{N}} k q_{s}^{*}(k)
$$

for the expected number of the children in $s$ generation of the process $\left(\left|B_{s}^{*}\right|\right)_{s \in \mathbb{N}}$.

Lemma 4.2.4 a) $\left|B_{s}^{*}\right| \leq\left|B_{s T, s T \theta}\right|$ for all $s \in \mathbb{N}$.

b) Suppose (4.1). Then,

$$
P^{\mathbf{q}}\left(B_{s}^{*} \neq \emptyset \text { for all } s \geq 1\right)>0 \text { Q-a.s. }
$$

and hence (4.2) holds.

Proof: a): This follows easily from (4.6) and induction on $s$.

b):By Theorem 1.1.2(a), it is enough to show that

$$
Q \ln m_{1}^{*}>0 \text { and } Q \ln \frac{1}{1-q_{1}^{*}(0)}<\infty .
$$


The first of the above can be seen as follows. Note that $B_{1}^{*}=B_{T, T \theta}$, and hence, by (4.10),

$$
m_{1}^{*}=P^{\mathbf{q}}\left[\left|B_{T, T \theta}\right|\right]=Z_{T, T \theta}
$$

Thus,

$$
Q\left[\ln m_{1}^{*}\right]=Q\left[\ln Z_{T, T \theta}\right]>0 .
$$

To see the second, we take $x_{0}, x_{1}, \ldots, x_{T}$ such that $x_{0}=0, x_{T}=\theta T$, and $\left|x_{t}-x_{t-1}\right|=1$, $t=1, \ldots, T$. Then,

$$
1-q_{1}^{*}(0)=P^{\mathbf{q}}\left(B_{T, T \theta} \neq \emptyset\right) \geq \prod_{t=0}^{T-1} \frac{1-q_{t, x_{t}}(0)}{2 d},
$$

Thus,

$$
Q \ln \frac{1}{1-q_{1}^{*}(0)} \leq T Q \ln \frac{1}{1-q_{0,0}(0)}+T \ln (2 d)<\infty
$$

End of the proof of Lemma 4.2.1; The condition (4.3) reads:

$$
Q\left[\frac{1}{m_{1}^{*}} \sum_{k \geq 1} k \ln k q_{1}^{*}(k)\right]<\infty .
$$

Thus, we have by Theorem 1.1 .2 (b) that

$$
\begin{aligned}
& \lim _{s \rightarrow \infty} \frac{1}{s} \ln \left|B_{s}^{*}\right|=\lim _{s \rightarrow \infty} \frac{1}{s} \sum_{u=1}^{s} \ln m_{u}^{*}=Q\left[\ln m_{1}^{*}\right], \\
& \text { a.s. on the event }\left\{B_{s}^{*} \neq \emptyset, \text { for all } s \geq 1\right\},
\end{aligned}
$$

where the second equality comes from the law of large numbers. Since

$$
\varliminf_{s \rightarrow \infty} \frac{1}{s T} \ln \left|B_{s T, s T \theta}\right| \geq \frac{1}{T} \lim _{s \rightarrow \infty} \frac{1}{s} \ln \left|B_{s}^{*}\right|
$$

and

$$
Q\left[\ln m_{1}^{*}\right]=Q \ln Z_{T, T \theta}>T \ln r
$$

we conclude the proof of Lemma 4.2.1 from (4.11).

\subsection{Proof of Theorem 2.1.1(b) and Proposition 2.2.2}

Since $\Psi=\psi(0)$, we get (2.4) from Lemma 4.2.2. To show (2.6), it is enough to prove the following lemma.

Lemma 4.3.1 Suppose that

$$
P\left(\varliminf_{s \rightarrow \infty} r^{-s T}\left|B_{s T}\right|>0\right)>0,
$$

for some $r>0$ and $T \in \mathbb{N}^{*}$ Then,

$$
\{\text { survival }\}=\left\{\varliminf_{t \rightarrow \infty} r^{-t}\left|B_{t}\right|>0\right\}, \quad P \text {-a.s. }
$$


Indeed, (2.6) follows easily from Lemma 4.2.1 and Lemma 4.3.1, In fact, let $r=\exp ((\Psi-\varepsilon))$. Then, since $\Psi=\psi(0)$, Lemma 4.2 .1 for $\theta=0$ implies (4.12) and hence (4.13) by Lemma 4.3 .1 .

Proof of Lemma 4.3.1: The following argument is adapted from [14, page 701]. We take $u \in\{0,1, \ldots, T-1\}$ and fix it for a moment. Then, by (4.12), the Markov property, and the shift invariance, we have:

$$
P\left(\varliminf_{s \rightarrow \infty} r^{-s T}\left|B_{s T+u}\right|>0\right)>0 .
$$

This implies that

$$
P\left(\inf _{s \geq 1} r^{-s T}\left|B_{s T+u}\right|>0\right)>0
$$

and hence that

1) $\quad \delta \stackrel{\text { def }}{=} P\left(\inf _{s \geq 1} r^{-s T}\left|B_{s T+u}\right|>\varepsilon\right)>0$ for some $\varepsilon>0$.

We now define a series of $\left(\mathcal{F}_{s T+u}\right)_{s \geq 1}$-stopping times $0=\sigma_{0}<\sigma_{1} \leq \sigma_{2} \leq \ldots$ as follows.

$$
\sigma_{1}=\inf \left\{s \geq 1 ; 1 \leq\left|B_{s T+u}\right| \leq \varepsilon r^{s T}\right\} .
$$

Note at this point that

$$
P\left(\sigma_{1}=\infty\right) \geq \delta
$$

thanks to (1). Suppose that $\sigma_{0}, \ldots, \sigma_{\ell}(\ell \geq 1)$ have already been defined. If $\sigma_{\ell}=\infty$, we set $\sigma_{n}=\infty$ for all $n \geq \ell+1$. Suppose that $\sigma_{\ell}<\infty$. Then $B_{\sigma_{\ell} T+u} \neq \emptyset$ and thus, there is a $z \in \mathbb{Z}^{d}$ such that

3) $B_{\sigma_{\ell} T+u, z} \neq \emptyset$

and for this $z$, there is a $\lambda \in \mathcal{T}_{\sigma_{\ell} T+u}$ such that

4) $(z, \lambda) \in B_{\sigma_{\ell} T+u}$.

Let $Z_{\ell}$ be the minimum, in the lexicographical order, of $z \in \mathbb{Z}^{d}$ such that (3) holds, and for $z=Z_{\ell}$, let $\lambda_{\ell}$ be the minimum, again in the lexicographical order, of $\lambda$ such that (4) holds. We now define $\sigma_{\ell+1}$ by:

$$
\sigma_{\ell+1}=\sigma_{\ell}+\inf \left\{s \geq 1 ; 1 \leq\left|B_{s T}^{\sigma_{\ell} T+u, Z_{\ell}, \lambda_{\ell}}\right| \leq \varepsilon r^{s T}\right\} .
$$

(Recall that $B^{s, z, \lambda}$ denotes the $(s, z, \lambda)$-branch of $B$.). It is easy to see from the construction that

5)

$$
P\left(\sigma_{\ell}<\infty \text { i.o. }\right)=0 \text {. }
$$

Indeed, we have

$$
P\left(\sigma_{\ell+1}<\infty \mid \mathcal{F}_{\sigma_{\ell} T+u}\right)=P\left(\sigma_{1}<\infty\right) \stackrel{(2)}{\leq} 1-\delta
$$

and hence

$$
\begin{aligned}
P\left(\sigma_{\ell+1}<\infty\right) & =P\left(\sigma_{\ell}<\infty, \sigma_{\ell+1}<\infty\right) \\
& =P\left(\sigma_{\ell}<\infty, P\left(\sigma_{\ell+1}<\infty \mid \mathcal{F}_{\sigma_{\ell} T+u}\right)\right) \\
& \leq(1-\delta) P\left(\sigma_{\ell}<\infty\right) \\
& \leq(1-\delta)^{\ell+1}
\end{aligned}
$$


by induction. Then, (5) follows from the Borel-Cantelli lemma.

By (5), we can pick a random $\ell \in \mathbb{N}$ such that almost surely, $\sigma_{\ell}<\infty$ and $\sigma_{\ell+1}=\infty$. Since $\sigma_{\ell}<\infty$, we have $B_{t+u} \supset B_{t-\sigma_{\ell} T}^{\sigma_{\ell} T+u, Z_{\ell}, \lambda_{\ell}}$ for all $t \geq \sigma_{\ell} T$. Note also that, on the event of survival, $\sigma_{\ell+1}=\infty$ implies that

$$
\left|B_{\left(s-\sigma_{\ell}\right) T}^{\sigma_{\ell} T+u, Z_{\ell}, \lambda_{\ell}}\right| \geq \varepsilon r^{\left(s-\sigma_{\ell}\right) T} \text { for } s \geq \sigma_{\ell} .
$$

Thus, on the event of survival,

$$
\left|B_{s T+u}\right| \geq\left|B_{\left(s-\sigma_{\ell}\right) T}^{\sigma_{\ell} T+u, Z_{\ell}, \lambda_{\ell}}\right| \geq \varepsilon r^{\left(s-\sigma_{\ell}\right) T} \text { for } s \geq \sigma_{\ell}
$$

hence

$$
\{\text { survival }\} \stackrel{\text { a.s. }}{\subset}\left\{\varliminf_{s \rightarrow \infty} r^{-s T}\left|B_{s T+u}\right|>0\right\} .
$$

Since the above is true for all $u=0,1, \ldots, T-1$ and

$$
\bigcap_{u=0}^{T-1}\left\{\underline{\lim }_{s \rightarrow \infty} r^{-s T}\left|B_{s T+u}\right|>0\right\}=\left\{\varliminf_{t \rightarrow \infty} r^{-t}\left|B_{t}\right|>0\right\},
$$

we get (4.13).

Proof of Proposition 2.2.2: We apply Lemma 4.3.1 to $T=1$ and $r=m$ to get the first equality. For the second one, we start to observe that the assumption implies that $P^{\mathbf{q}}\left(W_{\infty}\right)>$ 0 with positive $Q$-probability. By the zero-one law (e.g., cf. [9]), this event has $Q$-probability equal to 1 , and

$$
m^{-t} Z_{t}=P^{\mathbf{q}}\left[W_{t}\right] \rightarrow P^{\mathbf{q}}\left[W_{\infty}\right] \in(0, \infty), \quad Q \text {-a.s. }
$$

Writing $m^{-t}\left|B_{t}\right|=m^{-t} Z_{t} \times Z_{t}^{-1}\left|B_{t}\right|$, we derive the last equality.

\subsection{Local survival and growth rate at all large enough times}

In this subsection, we strengten Lemma 4.2.1 as follows to prepare the proof of Theorem $2.1 .2(\mathrm{~b})$ :

Lemma 4.4.1 Suppose $\theta \in \Delta \cap \mathbb{Q}^{d}$, (2.3) and that there exists $T \in \mathbb{N}^{*}(\theta)$ such that 4.1) holds. Then,

$$
P^{\mathbf{q}}\left(B_{t, t \theta} \neq \emptyset \text { for all large } t \in \mathbb{N}(\theta)\right)>0, \text { Q-a.s. }
$$

Suppose in addition that (4.3) holds. Then,

$$
P^{\mathbf{q}}\left(\left|B_{t, t \theta}\right| \geq e^{(\psi(\theta)-\varepsilon) t} \text { for all large } t \in \mathbb{N}^{*}(\theta)\right)>0, \quad Q \text {-a.s. }
$$

Proof: We will prove (4.14), mentioning at this point that (4.15) will be proved similarly. Recall notation $n(\theta)$ from (1.14). Fix $T=K n(\theta)$ such that (4.1) holds. Then, by Lemma 4.2.1,

$$
\alpha(\mathbf{q}) \stackrel{\text { def. }}{=} P^{\mathbf{q}}\left(B_{s T, s T \theta} \neq \emptyset \text { for all } s \geq 1\right)>0, \quad Q \text {-a.s. }
$$

We say that a family $\left(\mu^{(i)}, i \in I\right)$ (with $\left.\mu^{(i)} \in \mathcal{T}\right)$ is independent if for all $i \neq j \in I, \mu^{(i)}$ is not an ancestor of $\mu^{(j)}$. The reason for the terminology is that the branches $\left(B^{\left|\mu^{(i)}\right|, z^{(i)}, \mu^{(i)}} ; i \in I\right)$ are then independent for all choice of the $z^{(i)}$ 's. The idea of proof is that $Q$-a.s., due to branching, there is a positive probability to find a family of $K$ independent particles at times $(i+\ell K) n(\theta)$ and site $(i+\ell K) n(\theta) \theta \in \mathbb{Z}^{d}(i=1, \ldots, K)$. Then, by independence of the branches starting from these particles, $Q$-a.s., there is a positive probability for every such particle to generate a Smith-Wilkinson process in the direction $\theta$ which survives forever. 
We now write this in details. We have, with $\theta_{t, x}$ the time-space shift,

$$
P^{\mathbf{q}}\left(B_{s T, s T \theta}^{t, z, \mu} \neq \emptyset \text { for all } s \geq 1\right)=\alpha\left(\theta_{t, z} \mathbf{q}\right)>0
$$

We write $T(\ell, i)=(i+\ell K) n(\theta)$ to simplify the notation and let

$$
E=\left\{\left(\ell, \mu^{(1)}, \ldots, \mu^{(K)}\right) ; \ell \in \mathbb{N}, \mu^{(i)} \in \mathcal{T}_{T(\ell, i)},\left(\mu^{(i)}\right)_{i=1}^{K} \text { independent }\right\},
$$

and, for $\left(\ell, \mu^{(1)}, \ldots, \mu^{(K)}\right) \in E$, let

$$
\begin{aligned}
A\left(\ell, \mu^{(1)}, \ldots, \mu^{(K)}\right) & =\bigcap_{i=1}^{K}\left\{\left(T(\ell, i) \theta, \mu^{(i)}\right) \in B_{T(\ell, i)}\right\}, \\
S\left(\ell, \mu^{(1)}, \ldots, \mu^{(K)}\right) & =\bigcap_{i=1}^{K}\left\{B_{s T, s T \theta}^{T(\ell, i), T(\ell, i) \theta, \mu^{(i)}} \neq \emptyset \quad \forall s \geq 1\right\} .
\end{aligned}
$$

By independence,

$$
\begin{aligned}
P^{\mathbf{q}}\left(S\left(\ell, \mu^{(1)}, \ldots, \mu^{(K)}\right) \mid A\left(\ell, \mu^{(1)}, \ldots, \mu^{(K)}\right)\right) & =\prod_{i=1}^{K} \alpha\left(\theta_{T(\ell, i), T(\ell, i) \theta} \mathbf{q}\right) \\
& >0, \quad Q \text {-a.s. }
\end{aligned}
$$

by (4.16). Since

$$
\bigcup_{\left(\ell,\left(\mu^{(i)}\right)_{i=1}^{K}\right) \in E} A\left(\ell,\left(\mu^{(i)}\right)_{i=1}^{K}\right) \cap S\left(\ell,\left(\mu^{(i)}\right)_{i=1}^{K}\right) \subset\left\{B_{t, t \theta} \neq \emptyset \text { for all large } t \in \mathbb{N}^{*}(\theta)\right\},
$$

all what we need in order to prove our claim, is to show that the set of environments $\mathbf{q}$ such that

$$
P^{\mathbf{q}}\left(\bigcup_{\left(\ell, \mu^{(1)}, \ldots, \mu^{(K)}\right) \in E} A\left(\ell, \mu^{(1)}, \ldots, \mu^{(K)}\right)\right)>0
$$

has $Q$-probability 1. Observe that, since $\psi(\theta)>0$, we have $Q\left(q_{t, x}(0)+q_{t, x}(1) \leq 1-\epsilon\right)>0$ for some $\epsilon \in(0,1)$, and that $Q\left(q_{t, x}(0)<1\right)=1$ since $m>0$. Fix $S=\left(S_{i}\right)_{i \geq 0}$ a nearest neighbor path in $\mathbb{Z}^{d}$, with $S_{i n(\theta)}=i n(\theta) \theta, i=0,1, \ldots$. With overwhelming probability as $\ell$ increases, this path visits at least $K$ time-space sites where branching is possible in the first $\ell T$ steps:

$$
\lim _{\ell \rightarrow \infty} Q\left(C_{\ell}\right)=1
$$

with $C_{\ell} \subset \Omega_{\mathbf{q}}$ given by

$$
C_{\ell}=\left\{\sum_{i=0}^{\ell T-1} \mathbf{1}\left\{q_{i, S_{i}}(0)+q_{i, S_{i}}(1) \leq 1-\epsilon\right\} \geq K\right\} .
$$

Now, on $C_{\ell}$, the following scenario has positive $P^{\mathbf{q}}$-probability: the founding ancestor starts to follow the path $S$ giving birth to (at least) one child at each step, till the first branching site; it splits there into (at least) 2 particles, which continue to follow the path $S$ till the second branching site; there, the first one splits there into (at least) 2 particles, and these 3 particles continue till the next branching site, ...etc. To write this down precisely, we introduce the corresponding genealogies $\left(\nu^{(i)}\right)_{i=1}^{K}$, i.e.,

$$
\nu^{(i)} \in \mathcal{T}_{T(\ell, i)}
$$


composed of 1's only except for a 2 at the time of the $i$-th branching. Since $\left(\nu^{(i)}\right)_{i=1}^{K}$ are independent,

$$
\begin{aligned}
C_{\ell} & \subset\left\{\mathbf{q} \in \Omega_{q}: P^{\mathbf{q}}\left[A\left(\ell, \nu^{(1)}, \ldots, \nu^{(K)}\right)\right] \geq \epsilon^{K}(2 d)^{-(\ell+1) T} \prod_{i=0}^{(\ell+1) T-1}\left(1-q_{i, S_{i}}(0)\right)^{K}\right\} \\
& \subset\left\{\mathbf{q} \in \Omega_{q}: \text { (4.17) holds }\right\} .
\end{aligned}
$$

Hence,

$$
Q\left(P^{\mathbf{q}}\left(B_{t, t \theta} \neq \emptyset \text { for all large } t \in \mathbb{N}^{*}(\theta)\right)>0\right) \geq Q\left(C_{\ell}\right),
$$

which completes the proof of (2.12). The proof of (2.14) is totally similar, but using the second statement of Lemma 4.2.1,

\subsection{Proof of Theorem 2.1.2(b)}

Proof of (2.12): By Lemma 4.4.1, we have

$$
P^{\mathbf{q}}\left(B_{t, t \theta} \neq \emptyset \text { for all large } t \in \mathbb{N}^{*}(\theta)\right)>0, \quad Q \text {-a.s. }
$$

This means that $Q$-a.s. we can find a time $u=u(\mathbf{q}) \in \mathbb{N}^{*}(\theta)$ and a genealogy $\nu=\nu(\mathbf{q}) \in \mathcal{T}_{u}$ such that

1)

$$
P^{\mathbf{q}}\left(\left\{(u \theta, \nu) \in B_{u}\right\} \cap F_{\nu}\right)>0 \quad Q \text {-a.s. }
$$

where

$$
F_{\lambda}=\left\{B_{t,(t+u) \theta}^{u, u \theta, \lambda} \neq \emptyset \text { for all } t \in \mathbb{N}^{*}(\theta)\right\} \quad \text { for any } \lambda \in \mathcal{T}_{u} .
$$

We now take a nearest neighbor path $S=\left(S_{t}\right)_{t=0}^{u}$ in $\mathbb{Z}^{d}$ such that $S_{t}=t \theta$ for all $t \in$ $\mathbb{N}(\theta) \cap[0, u]$, and fix it. Let $\mu=(1, \ldots, 1) \in \mathcal{T}_{u}$ and

$$
E_{\mu, S}=\left\{\text { The genealogy } \mu \text { follows }\left(S_{t}\right)_{t=0}^{u} \text { and } K_{t, S_{t}, \mu} \geq 1 \text { for all } t=0, \ldots, u-1\right\} .
$$

Note that $E_{\mu, S}$ and $F_{\mu}$ are independent under $P^{\mathbf{q}}$, since

$$
E_{\mu, S} \in \sigma\left(\left(X_{t, \cdot, \mu}, K_{t, \cdot, \mu}\right)_{t \leq u-1}\right), \quad F_{\mu} \in \sigma\left(\left(X_{t, \cdot,}, K_{t, \cdot,}\right)_{t \geq u}\right) .
$$

Moreover,

$$
\begin{aligned}
P^{\mathbf{q}}\left(E_{\mu, S}\right) & =\prod_{t=0}^{u-1}\left(\frac{1-q_{t, S_{t}}(0)}{2 d}\right)>0, \\
P^{\mathbf{q}}\left(F_{\mu}\right) & =P^{\mathbf{q}}\left(F_{\nu}\right) \stackrel{(1)}{>} 0 .
\end{aligned}
$$

Therefore, $Q$-a.s.

$$
P^{\mathbf{q}}\left(B_{t, t \theta} \neq \emptyset \text { for all } t \in \mathbb{N}(\theta)\right) \geq P^{\mathbf{q}}\left(E_{\mu, S} \cap F_{\mu}\right)=P^{\mathbf{q}}\left(E_{\mu, S}\right) P^{\mathbf{q}}\left(F_{\mu}\right)>0 .
$$

Proof of 2.14): By Lemma 4.4.1 again, we have

$$
P^{\mathbf{q}}\left(\left|B_{t, t \theta}\right| \geq e^{(\psi(\theta)-\varepsilon) t} \text { for all large } t \in \mathbb{N}(\theta)\right)>0 \quad Q \text {-a.s. }
$$

This, together with the similar argument as the proof of (2.12) implies that

$$
P^{\mathbf{q}}\left(\inf _{t \in \mathbb{N}(\theta)} e^{-(\psi(\theta)-\varepsilon) t}\left|B_{t, t \theta}\right|>0\right)>0 \quad Q \text {-a.s. }
$$

Then, (2.14) follows from the same argument as Lemma 4.3.1. 


\subsection{Generating function of BRWRE}

For $q \in \mathcal{P}(\mathbb{N})$, we define its generating function by

$$
\widehat{q}(s)=\sum_{k \geq 0} s^{k} q(k) \quad s \in[0,1]
$$

Here and in what follows, we agree that $0^{0}=1$. For a fixed $\mathbf{q} \in \Omega_{\mathbf{q}}$ and $t \in \mathbb{N}$, we define $\Phi_{t}:[0,1]^{\mathbb{Z}^{d}} \rightarrow[0,1]^{\mathbb{Z}^{d}}$ by

$$
\Phi_{t}(\xi)=\left(\Phi_{t, x}(\xi)\right)_{x \in \mathbb{Z}^{d}}, \quad \Phi_{t, x}(\xi)=\sum_{y \in \mathbb{Z}^{d}} p(x, y) \widehat{q}_{t, x}\left(\xi_{y}\right)
$$

Hence $\left(\Phi_{t}\right)_{t \in \mathbb{N}}$ is a sequence of i.i.d. random maps on the probability space $\left(\Omega_{\mathbf{q}}, \mathcal{F}_{\mathbf{q}}, Q\right)$.

Lemma 4.6.1 For $\xi \in[0,1]^{\mathbb{Z}^{d}}$ and $t \in \mathbb{N}^{*}$,

$$
P^{\mathrm{q}}\left[\xi^{B_{t}} \mid \mathcal{F}_{t-1}\right]=\Phi_{t-1}(\xi)^{B_{t-1}}
$$

with the notation

$$
\xi^{B_{t}}=\prod_{x \in \mathbb{Z}^{d}} \xi_{x}^{\left|B_{t, x}\right|} \in[0, \infty)
$$

As a consequence,

$$
P^{\mathbf{q}}\left[\xi^{B_{t}}\right]=\Phi_{0,0} \circ \Phi_{1} \circ \ldots \circ \Phi_{t-1}(\xi) .
$$

Proof: It is enough to show (4.20). We begin by writing:

$$
\begin{aligned}
\xi^{B_{t}}=\prod_{y \in \mathbb{Z}^{d}} \xi_{y}^{\left|B_{t, y}\right|} & =\prod_{y \in \mathbb{Z}^{d}} \prod_{(x, \nu) \in B_{t-1}} \xi_{y}^{K_{t-1, x, \nu} \delta_{y}\left(X_{t-1, x, \nu}\right)}=\prod_{(x, \nu) \in B_{t-1}} \prod_{y \in \mathbb{Z}^{d}} \xi_{y}^{K_{t-1, x, \nu} \delta_{y}\left(X_{t-1, x, \nu}\right)} \\
& =\prod_{(x, \nu) \in B_{t-1}} \prod_{y \in \mathbb{Z}^{d}}\left(1+\left(\xi_{y}^{K_{t-1, x, \nu}}-1\right) \delta_{y}\left(X_{t-1, x, \nu}\right)\right) \\
& =\prod_{(x, \nu) \in B_{t-1}}\left(1+\sum_{y \in \mathbb{Z}^{d}}\left(\xi_{y}^{K_{t-1, x, \nu}}-1\right) \delta_{y}\left(X_{t-1, x, \nu}\right)\right) \\
& =\prod_{(x, \nu) \in B_{t-1}} \sum_{y \in \mathbb{Z}^{d}} \xi_{y}^{K_{t-1, x, \nu}} \delta_{y}\left(X_{t-1, x, \nu}\right)
\end{aligned}
$$

where on the third line, we have used that

1)

$$
\prod_{y \in Y}\left(1+x_{y}\right)=1+\sum_{A \subset Y} \prod_{y \in A} x_{y} \quad \text { for any finite set } Y \text { and }\left(x_{y}\right)_{y \in Y} \in \mathbb{R}^{Y}
$$

(the terms with $|A| \geq 2$ on the right-hand-side of (1) vanishes in our application). Since

$$
P^{\mathbf{q}}\left[\sum_{y \in \mathbb{Z}^{d}} \xi_{y}^{K_{t-1, x, \nu}} \delta_{y}\left(X_{t-1, x, \nu}\right) \mid \mathcal{F}_{t-1}\right]=\Phi_{x, t-1}(\xi),
$$

We get

$$
P^{\mathbf{q}}\left[\xi^{B_{t}} \mid \mathcal{F}_{t-1}\right]=\prod_{(x, \nu) \in B_{t-1}} \Phi_{x, t-1}(\xi)=\Phi_{t-1}(\xi)^{B_{t-1}}
$$


Proof of Proposition 2.2.1: We note that the map $\Phi_{t}(t \in \mathbb{N})$ has the following continuity property:

$$
\xi, \xi_{n} \in[0,1]^{\mathbb{Z}^{d}} \lim _{n} \xi_{n}=\xi \Longrightarrow \lim _{n} \Phi_{t}\left(\xi_{n}\right)=\Phi_{t}(\xi),
$$

where the limits are coordinatewise. We have also that

$$
\Phi_{0,0} \circ \Phi_{1} \circ \ldots \circ \Phi_{t-1}(\xi)=1 \Longleftrightarrow \xi_{x}=1 \text { for all } x \in \mathbb{Z}^{d} \text { with } P_{S}\left(S_{t}=x\right)>0 .
$$

Let $B^{x}$ be the BRWRE starting from one particle from time-space $(0, x) \in \mathbb{N} \times \mathbb{Z}^{d}$. Then,for the zero-field $\xi$ (i.e., $\xi$. $\equiv 0$ ),

$$
\delta_{t, x}(\mathbf{q}) \stackrel{\text { def }}{=} P^{\mathbf{q}}\left(B_{t}^{x}=\emptyset\right)=P^{\mathbf{q}}\left[\xi^{B_{t}^{x}}\right] \stackrel{(4.21)}{=} \Phi_{0, x} \circ \Phi_{1} \circ \ldots \circ \Phi_{t-1}(\xi) .
$$

Since $\delta_{t, x}(\mathbf{q})$ is decreasing in $t$, the limit $\delta_{x}(\mathbf{q})=\lim _{t \rightarrow \infty} \delta_{t, x}(\mathbf{q})$ exists for all $x \in \mathbb{Z}^{d}$ and we define the random field of extinction probabilities:

$$
\delta(\mathbf{q})=\left(\delta_{x}(\mathbf{q})\right)_{x \in \mathbb{Z}^{d}} .
$$

Note that $\delta_{0}(\mathbf{q})$ is the extinction probability of $B_{t}$ :

$$
\delta_{0}(\mathbf{q})=1-P^{\mathbf{q}}(\text { survival }) .
$$

Now, we have for any $u \in \mathbb{N}^{*}$ that

$$
\begin{array}{rll}
\delta(\mathbf{q}) & \stackrel{(1)}{=} & \lim _{t \rightarrow \infty} \Phi_{0} \circ \Phi_{1} \circ \ldots \circ \Phi_{t-1}(\xi) \\
& \stackrel{(4.22)}{=} & \Phi_{0} \circ \Phi_{1} \circ \ldots \circ \Phi_{u-1}\left(\lim _{t \rightarrow \infty} \Phi_{u} \circ \ldots \circ \Phi_{u+t-1}\right) \\
& \stackrel{(1)}{=} \quad \Phi_{0} \circ \Phi_{1} \circ \ldots \circ \Phi_{u-1}\left(\delta\left(\theta_{u, 0} \mathbf{q}\right)\right),
\end{array}
$$

where $\theta_{u, y}$ denotes the shift: $\theta_{u, y} \mathbf{q}=\left(q_{\cdot+u, \cdot+y}\right)$. This and (4.23) imply that

$$
\left\{\mathbf{q} ; \delta_{0}(\mathbf{q})=1\right\}=\bigcap_{u \geq 1}\left\{\mathbf{q} ; \delta_{x}\left(\theta_{u} \mathbf{q}\right)=1 \text { for all } x \in \mathbb{Z}^{d} \text { with } P_{S}\left(S_{t}=x\right)>0\right\}
$$

Since the right-hand-side is a tail event, it is $Q$-trivial by Kolmogorov's zero-one law.

In analogy with GW and SW processes, the extinction probability of BRWRE can be characterized in terms of the functional equation involving the generating function. To explain it, we introduce the coordinatewise order in $[0,1]^{\mathbb{Z}^{d}}$. Let $\xi, \eta \in[0,1]^{\mathbb{Z}^{d}}$. We write $\xi \leq \eta$ when $\xi_{x} \leq \eta_{x}$ for all $x \in \mathbb{Z}^{d}$. A function $F:[0,1]^{\mathbb{Z}^{d}} \rightarrow[0,1]^{\mathbb{Z}^{d}}$ is called increasing if it preserves this order.

Corollary 4.6.2 The random field $\delta(\mathbf{q})$ defined by (4.24) is the minimal among all random fields $\xi(\mathbf{q})$ such that

$$
\xi(\mathbf{q})=\Phi_{0}\left(\xi\left(\theta_{1,0} \mathbf{q}\right)\right) .
$$

Moreover, the extinction probability $\delta_{0}(\mathbf{q})$ (cf. (4.25)) is the minimal $[0,1]$-valued function $\xi_{0}(\mathbf{q})$ of $\mathbf{q} \in \mathcal{P}(\mathbb{N})^{\mathbb{N} \times \mathbb{Z}^{d}}$ such that

$$
\xi_{0}(\mathbf{q})=\Phi_{0,0}\left(\left(\xi\left(\theta_{1, x} \mathbf{q}\right)_{x \in \mathbb{Z}^{d}}\right)\right) .
$$


Proof: By setting $u=1$ in (4.26), we see that $\delta(\mathbf{q})$ is indeed a solution of (4.27). On the other hand, any solution $\xi(\mathbf{q})$ of (4.27) satisfies:

$$
\begin{aligned}
\xi(\mathbf{q}) & =\Phi_{0}\left(\xi\left(\theta_{1,0} \mathbf{q}\right)\right)=\Phi_{0} \circ \Phi_{1}\left(\xi\left(\theta_{2,0} \mathbf{q}\right)\right)=\ldots \\
& =\lim _{t \rightarrow \infty} \Phi_{0} \circ \ldots \circ \Phi_{t-1}\left(\xi\left(\theta_{t, 0} \mathbf{q}\right)\right) .
\end{aligned}
$$

Comparing this with the definition of $\delta(\mathbf{q})$, we see that $\delta(\mathbf{q}) \leq \xi(\mathbf{q})$, since $\Phi_{u}, u \in \mathbb{N}$ are increasing. These prove the first half of the corollary.

A function $\xi_{0}: \mathcal{P}(\mathbb{N})^{\mathbb{N} \times \mathbb{Z}^{d}} \rightarrow[0,1]$ solves (4.28) if and only if the random field defined by $\xi(\mathbf{q})=\left(\xi_{0}\left(\theta_{0, x} \mathbf{q}\right)\right)_{x \in \mathbb{Z}^{d}}$ solves (4.27). Thus, the second half of the corollary comes down to the first half.

We next turn to the proof of (2.9) .

Lemma 4.6.3 Let $q(k)=Q\left[q_{0,0}(k)\right], k \in \mathbb{N}$. Then, for $s \in[0,1]$,

$$
P\left[s^{\left|B_{t}\right|}\right] \geq(\underbrace{\widehat{q} \circ \widehat{q} \circ \ldots \circ \widehat{q}}_{t})(s) .
$$

Proof: It follows from (4.20) and Hölder's inequality that

$$
P\left[s^{\left|B_{t}\right|} \mid \mathcal{F}_{t-1}\right]=\prod_{x \in \mathbb{Z}^{d}} Q\left[\widehat{q}_{0,0}(s)^{n}\right]_{n=\left|B_{t-1, x}\right|} \geq \prod_{x \in \mathbb{Z}^{d}} Q\left[\widehat{q}_{0,0}(s)\right]^{\left|B_{t-1, x}\right|}=\widehat{q}(s)^{\left|B_{t-1}\right|} .
$$

Thus, (4.29) follows by iteration.

Lemma 4.6.4 For $s \in[0,1]$,

$$
P\left[s^{\left|B_{t}\right|}\right] \leq Q\left[\widehat{q}_{0,0} \circ \widehat{q}_{1,0} \circ \ldots \circ \widehat{q}_{t-1,0}(s)\right] .
$$

Proof: We will prove by induction that for $1 \leq u \leq t$,

$$
P\left[s^{\left|B_{t}\right|} \mid \mathcal{F}_{t-u}\right] \leq Q\left[\left(\widehat{q}_{0,0} \circ \widehat{q}_{1,0} \circ \ldots \circ \widehat{q}_{u-1,0}\right)(s)^{n}\right]_{n=\left|B_{t-u}\right|} .
$$

This in particular proves (4.30) $(u=t)$. It follows from (4.20) and Hölder's inequality that

$$
P\left[s^{\left|B_{t}\right|} \mid \mathcal{F}_{t-1}\right]=\prod_{x \in \mathbb{Z}^{d}} Q\left[\widehat{q}_{0,0}(s)^{n}\right]_{n=\left|B_{t-1, x}\right|} \leq Q\left[\widehat{q}_{0,0}(s)^{n}\right]_{n=\left|B_{t-1}\right|},
$$

which proves (1) for $u=1$. Now assume that (1) with $u$ replaced by $u-1(u \geq 2)$ is true and let $A \in \mathcal{F}_{t-u}$. Then, by the assumption and Fubini's theorem,

$$
\begin{aligned}
E\left[s^{\left|B_{t}\right|} \mathbf{1}_{A}\right] & \leq P\left[\mathbf{1}_{A} Q\left[\left(\widehat{q}_{0,0} \circ \widehat{q}_{1,0} \circ \ldots \circ \widehat{q}_{u-2,0}\right)(s)^{n}\right]_{n=\left|B_{t-u+1}\right|}\right] \\
& =Q\left[P\left[\mathbf{1}_{A}\left(\widehat{q}_{t-u+1,0} \circ \widehat{q}_{t-u+2,0} \circ \ldots \circ \widehat{q}_{t-1,0}\right)(s)^{\left|B_{t-u+1}\right|}\right]\right] .
\end{aligned}
$$

By (1) with $u=1$, we have that

$$
\begin{aligned}
P & {\left[\mathbf{1}_{A}\left(\widehat{q}_{t-u+1,0} \circ \widehat{q}_{t-u+2,0} \circ \ldots \circ \widehat{q}_{t-1,0}\right)(s)^{\left|B_{t-u+1}\right|}\right] } \\
& \leq P\left[\mathbf{1}_{A} \int Q\left(d q_{t-u, 0}\right)\left(\widehat{q}_{t-u, 0} \circ \widehat{q}_{t-u+1,0} \circ \ldots \circ \widehat{q}_{t-1,0}\right)(s)^{\left|B_{t-u}\right|}\right] .
\end{aligned}
$$

Here, $\int Q\left(d q_{t-u, 0}\right)$ means that we only integrate $q_{t-u, 0}$, with all the other $q_{., \text {. fixed. Combining }}$ these, we arrive at

$$
E\left[s^{\left|B_{t}\right|} \mathbf{1}_{A}\right] \leq P\left[\mathbf{1}_{A} Q\left[\left(\widehat{q}_{t-u, 0} \circ \widehat{q}_{t-u+1,0} \circ \ldots \circ \widehat{q}_{t-1,0}\right)(s)^{n}\right]_{n=\left|B_{t-u}\right|}\right],
$$


which proves (1).

Proof of (2.9): Let $\left(B_{t}^{\mathrm{GW}}\right)_{t \in \mathbb{N}}$ and $\left(B_{t}^{\mathrm{SW}}\right)_{t \in \mathbb{N}}$ be the Galton-Watson and Smith-Wilkinson processes we are interested in. Then, for $s \in[0,1]$,

$$
\begin{aligned}
P\left[s^{\left|B_{t}^{\mathrm{GW}}\right|}\right] & =(\underbrace{\widehat{q} \circ \widehat{q} \circ \ldots \circ \widehat{q}}_{t})(s), \\
P\left[s^{\left|B_{t}^{\mathrm{SW}}\right|}\right] & =Q\left[\widehat{q}_{0,0} \circ \widehat{q}_{1,0} \circ \ldots \circ \widehat{q}_{t-1,0}(s)\right] .
\end{aligned}
$$

The former is well-known and for the latter, see [23, Theorem 2.1]. For $s=0$, we have

$$
P(\text { survival })=1-\lim _{t \rightarrow \infty} P\left[s^{\left|B_{t}\right|}\right] .
$$

and similar formulae for $\sigma^{\mathrm{GW}}$ and $\sigma^{\mathrm{SW}}$. Therefore, (2.9) follows from Lemma 4.6.3 and Lemma 4.6.4.

\section{Appendix}

\subsection{Proof of Proposition 3.2.1(a)}

If $m_{0,0} \equiv m$ a.s., then $\psi(\theta)=\ln m-I_{S}(\theta)$. Thus, we assume that $m_{0,0} \not \equiv m$ a.s. We start by proving the following.

a) For $\theta \in \Delta \cap \mathbb{Q}^{d}$ and $t \in \mathbb{N}^{*}(\theta)$,

$$
\psi(\theta) \geq \frac{1}{t} Q\left[\ln Z_{t, t \theta}\right] \geq Q\left[\ln m_{0,0}\right]+\frac{1}{t} \ln P_{S}\left(S_{t}=t \theta\right) .
$$

Moreover, the second inequality is an equality if and only if $t=1$ or $\theta \in\left\{ \pm e_{i}\right\}_{i=1}^{d}$.

b) For $t \geq 1$,

$$
\psi(0) \geq \frac{1}{t} Q\left[\ln Z_{t}\right] \geq Q\left[\ln m_{0,0}\right] .
$$

Moreover, the second inequality is an equality if and only if $t=1$.

These can be seen as follows:

$$
\begin{aligned}
Z_{t, t \theta} & =P_{S}\left[\prod_{u=0}^{t-1} m_{u, S_{u}} \mid S_{t}=t \theta\right] P_{S}\left(S_{t}=t \theta\right) \\
& \stackrel{\operatorname{Jensen}}{\geq} \exp \left(\sum_{u=0}^{t-1} P_{S}\left[\ln m_{u, S_{u}} \mid S_{t}=t \theta\right]\right) P_{S}\left(S_{t}=t \theta\right)
\end{aligned}
$$

Note that, if $t \neq 1$ and $\theta \notin\left\{ \pm e_{i}\right\}_{i=1}^{d}$ the random variable $S_{1}$ under $P_{S}\left(\cdot \mid S_{t}=t \theta\right)$ is not a constant. Thus, the Jensen inequality above is strict on the event:

$$
\left\{\mathbf{q} ; m_{1, e} \neq m_{1, e^{\prime}} \text { for some } e, e^{\prime} \in \mathbb{Z}^{d} \text { with }|e|=\left|e^{\prime}\right|=1\right\},
$$

which has positive $Q$-probability by the assumption. By taking logarithm, and then $Q$ expectation, we get the second inequality of (5.1). On the other hand, it is not difficult to 
prove that the sequence $\left\{Q\left[\ln Z_{t, t \theta}\right]\right\}_{t \in \mathbb{N}^{*}}$ is superadditive (e.g., 9 , page 720, Proof of Proposition 2.5]) and hence

$$
\psi(\theta)=\sup _{t \geq 1} \frac{1}{t} Q\left[\ln Z_{t, t \theta}\right]
$$

This completes the proof of of (5.1). The proof of (5.2) is similar.

By letting $t \rightarrow \infty$ in (5.1), we get

$$
\psi(\theta) \geq Q\left[\ln m_{0,0}\right]-I_{S}(\theta) .
$$

Finally, we prove

$$
\psi(\theta) \leq \ln m-I_{S}(\theta)
$$

We have

$$
\begin{aligned}
& Q\left[\ln Z_{t, t \theta}\right]=Q\left[\ln P_{S}\left[\prod_{u=0}^{t-1} m_{u, S_{u}}: S_{t}=t \theta\right]\right] \\
& \stackrel{\text { Jensen }}{\leq} \ln Q\left[P_{S}\left[\prod_{u=0}^{t-1} m_{u, S_{u}}: S_{t}=t \theta\right]\right]=t \ln m+\ln P_{S}\left(S_{t}=t \theta\right)
\end{aligned}
$$

We get the desired bound by dividing the above inequality by $t$ and then letting $t \rightarrow \infty$.

\subsection{Proof of Proposition 3.2 .1 (b)}

Let $(\Omega, \mathcal{G}, Q)$ be a probability space and

$$
\{\emptyset, \Omega\}=\mathcal{G}_{0} \subset \mathcal{G}_{1} \subset \ldots
$$

be an increasing series of sub $\sigma$-fields of $\mathcal{G}$. For $X \in L^{1}(Q)$, we write $Q^{\mathcal{G}_{j}}[X]=Q\left[X \mid \mathcal{G}_{j}\right]$. We use a concentration inequality in the following Lemma.

Lemma 5.2.1 Suppose that $X \in L^{1}(Q)$ is $\mathcal{G}_{n}$-measurable for some $n$ and that there exist $\delta \in(0, \infty), A \in[0, \infty), X_{1}, \ldots, X_{n} \in L^{1}(Q)$ such that

$$
Q^{\mathcal{G}_{j-1}}\left[X_{j}\right]=Q^{\mathcal{G}_{j}}\left[X_{j}\right] \text {, and } Q^{\mathcal{G}_{j-1}}\left[\exp \left(\delta\left|X-X_{j}\right|\right)\right] \leq A
$$

for all $j=1, \ldots, n$. Then, with $B=2 \sqrt{6} A^{2} / \delta^{2}$,

$$
Q(|X-Q[X]| \geq \varepsilon n) \leq 2 \exp \left(-B \varepsilon^{2} n / 4\right) \text { for all } \varepsilon \in[0, B \delta] .
$$

Proof: We consider a sequence $D_{j}=Q^{\mathcal{G}_{j}}[X]-Q^{\mathcal{G}_{j-1}}[X]$. We first observe that

1) $\quad Q^{\mathcal{G}_{j-1}}\left[e^{\delta\left|D_{j}\right|}\right] \leq A^{2}$ for all $j=1, \ldots, n$.

Since $Q^{\mathcal{G}_{j-1}}\left[X_{j}\right]=Q^{\mathcal{G}_{j}}\left[X_{j}\right]$, we have

$$
\begin{aligned}
\left|D_{j}\right| & \leq\left|Q^{\mathcal{G}_{j}}\left[X-X_{j}\right]\right|+\left|Q^{\mathcal{G}_{j-1}}\left[X-X_{j}\right]\right| \\
& \leq Q^{\mathcal{G}_{j}}\left[Y_{j}\right]+Q^{\mathcal{G}_{j-1}}\left[Y_{j}\right], \text { with } Y_{j}=\left|X-X_{j}\right| .
\end{aligned}
$$

It follows from Jensen inequality that

$$
e^{\delta Q^{\mathcal{G}_{j-1}}\left[Y_{j}\right]} \leq Q^{\mathcal{G}_{j-1}}\left[e^{\delta Y_{j}}\right] \leq A .
$$

Similarly,

$$
Q^{\mathcal{G}_{j-1}}\left[e^{\delta Q^{\mathcal{G}_{j}}\left[Y_{j}\right]}\right] \leq Q^{\mathcal{G}_{j-1}}\left[Q^{\mathcal{G}_{j}}\left[e^{\delta Y_{j}}\right]\right]=Q^{\mathcal{G}_{j-1}}\left[e^{\delta Y_{j}}\right] \leq A .
$$

These imply (1) as follows:

$$
Q^{\mathcal{G}_{j-1}}\left[e^{\delta\left|D_{j}\right|}\right] \leq e^{\delta Q^{\mathcal{G}_{j-1}}\left[Y_{j}\right]} Q^{\mathcal{G}_{j-1}}\left[e^{\delta Q^{\mathcal{G}_{j}}\left[Y_{j}\right]}\right] \leq A^{2} .
$$

We now infer from (1) that 
2)

$$
Q^{\mathcal{G}_{j-1}}\left[e^{\alpha D_{j}}\right] \leq e^{B \alpha^{2}} \text { for all } \alpha \in[-\delta / 2, \delta / 2] \text { and } j=1, \ldots, n .
$$

Observe that

$$
\frac{1}{4 !} Q^{\mathcal{G}_{j-1}}\left[\left|D_{j}\right|^{4}\right]=\frac{1}{4 !} Q^{\mathcal{G}_{j-1}}\left[\delta^{4}\left|D_{j}\right|^{4}\right] / \delta^{4} \leq Q^{\mathcal{G}_{j-1}}\left[e^{\delta\left|D_{j}\right|}\right] / \delta^{4} \leq A^{2} / \delta^{4}
$$

and hence that

$$
Q^{\mathcal{G}_{j-1}}\left[\left|D_{j}\right|^{2} e^{\delta\left|D_{j}\right| / 2}\right] \leq Q^{\mathcal{G}_{j-1}}\left[\left|D_{j}\right|^{4}\right]^{1 / 2} Q^{\mathcal{G}_{j-1}}\left[e^{\delta\left|D_{j}\right|}\right]^{1 / 2} \leq 2 \sqrt{6} A^{2} / \delta^{2}=B .
$$

Since $e^{x} \leq 1+x+|x|^{2} e^{|x|} / 2$ for all $x \in \mathbb{R}$, we get

$$
Q^{\mathcal{G}_{j-1}}\left[e^{\alpha D_{j}}\right] \leq 1+\alpha^{2} B / 2 \leq \exp \left(B \alpha^{2}\right)
$$

Finally, since $X-Q[X]=D_{n}+\ldots+D_{1}$, it follows from (2) that

$$
Q[\exp (\alpha(X-Q[X]))] \leq \exp \left(B \alpha^{2} n\right) \text { for all } \alpha \in[-\delta / 2, \delta / 2]
$$

via a simple iterative procedure. To see (5.4), we take $\alpha=\frac{\varepsilon}{2 B} \leq \frac{\delta}{2}$. Then, by Chebychev's inequality and (3),

$$
\begin{aligned}
Q(|X-Q[X]| \geq \varepsilon n) & =Q(\alpha|X-Q[X]| \geq \alpha \varepsilon n) \\
& \leq 2 \exp \left(\left(B \alpha^{2}-\alpha \varepsilon\right) n\right)=2 \exp \left(-B \varepsilon^{2} n / 4\right) .
\end{aligned}
$$

Proof of Proposition 3.2.1(b): We fix an arbitrary integer $t$ and set $\mathcal{G}_{j}=\sigma\left(m_{k,}: k \leq j\right)$ $(j=1, \ldots, t)$. To prove (3.16) and (3.15) at a stroke, we define

$$
X=\ln P_{S}\left[\zeta_{t} f\left(S_{t}\right)\right],
$$

where $f: \mathbb{Z}^{d} \rightarrow[0, \infty)$ is such that $P_{S}\left[f\left(S_{t}\right)\right]>0 . X=\ln Z_{t, t \theta}$ for $f(x)=\delta_{x, t \theta}$ and, $X=\ln Z_{t}$ for $f(x) \equiv 1$. We will prove that $X$ satisfies (5.4) with some $B$, independent of the choice of $f$. To do so, let

$$
X_{j}=\ln P_{S}\left[\zeta_{t, j} f\left(S_{t}\right)\right], \quad j=1, \ldots, t,
$$

where

$$
\zeta_{t, j}=\prod_{\substack{0 \leq k \leq t-1 \\ k \neq j}} m_{k, S_{k}} .
$$

We then check (5.3). Since $X_{j}$ does not depend on $m_{\cdot, j}$, we have $Q^{\mathcal{G}_{j-1}}\left[X_{j}\right]=Q^{\mathcal{G}_{j}}\left[X_{j}\right]$. Note on the other hand that for $\delta \in \mathbb{R} \backslash(0,1)$,

$$
\exp \left(\delta\left(X-X_{j}\right)\right)=\left(\frac{P_{S}\left[\zeta_{t} f\left(S_{t}\right)\right]}{P_{S}\left[\zeta_{t, j} f\left(S_{t}\right)\right]}\right)^{\delta}=\frac{\left(\frac{P_{S}\left[\zeta_{t, j} m_{j, S_{j}} f\left(S_{t}\right)\right]}{P_{S}\left[\zeta_{t, j} f\left(S_{t}\right)\right]}\right)^{\delta}}{\stackrel{\substack{\text { Jensen } \\ \leq}}{\frac{P_{S}\left[\zeta_{t, j} m_{j, S_{j}}^{\delta} f\left(S_{t}\right)\right]}{P_{S}\left[\zeta_{t, j} f\left(S_{t}\right)\right]}}}
$$

Now, by taking the conditional expectation given $\mathcal{G}_{j}^{\prime} \stackrel{\text { def. }}{=} \sigma\left[m_{k,} ; k \neq j\right]$,

$$
Q^{\mathcal{G}_{j}^{\prime}}\left[\exp \left(\delta\left(X-X_{j}\right)\right)\right] \leq Q\left[m_{0,0}^{\delta}\right] .
$$

This, together with $\mathcal{G}_{j-1} \subset \mathcal{G}_{j}^{\prime}$, implies

$$
Q^{\mathcal{G}_{j-1}}\left[\exp \left(\delta\left(X-X_{j}\right)\right)\right] \leq Q\left[m_{0,0}^{\delta}\right] .
$$

By applying this for $\delta= \pm 1$, we get

$$
Q^{\mathcal{G}_{j-1}}\left[\exp \left(\left|X-X_{j}\right|\right)\right] \leq A:=Q\left[m_{0,0}+m_{0,0}^{-1}\right] .
$$




\section{References}

[1] Athreya, K.; Karlin, S.: Branching processes with random environments. I. Extinction probabilities. Ann. Math. Statist. 42 (1971), 1499-1520.

[2] Athreya, K.; Karlin, S.: Branching processes with random environments. II. Limit theorems. Ann. Math. Statist. 42 (1971), 1843-1858.

[3] Athreya, K. and Ney, P. (1972): Branching Processes, Springer Verlag New York.

[4] Biggins, J. (1979): Growth rates in the branching random walk. Z. Wahrsch. Verw. Gebiete 48 (1979) 17-34.

[5] Birkner, M.(2003): Particle systems with locally dependent branching: long-time behaviour, genealogy and critical parameters. PhD thesis, Johann Wolfgang Goethe-Universität, Frankfurt.

[6] Birkner, M., Geiger, J., Kersting G.(2005): Branching processes in random environment- a view on critical and subcritical cases. Interacting stochastic systems, 269-291, Springer, Berlin.

[7] Carmona, P., Hu Y.: Fluctuation exponents and large deviations for directed polymer in a random environment, Stoch. Proc. Appl. 112, (2004), 285-308.

[8] Comets, F., Popov, S.: Shape and local growth for multidimensional branching random walks in random environment. ALEA Lat. Am. J. Probab. Math. Stat. 3 (2007), 273-299

[9] Comets, F., Shiga, T., Yoshida, N.(2003) Directed Polymers in Random Environment: Path Localization and Strong Disorder, Bernoulli 9, 705-723.

[10] Comets, F., Shiga, T., Yoshida, N. (2004) Probabilistic analysis of directed polymers in random environment: a review, Advanced Studies in Pure Mathematics, 39, 115-142.

[11] Comets, F., Vargas, V. (2004) Majorizing multiplicative cascades for directed polymers in random media. ALEA Lat. Am. J. Probab. Math. Stat. 2, 267-277.

[12] Durrett, R. (2005):"Probability-Theory and Examples", 3rd Ed., Brooks/Cole-Thomson Learning.

[13] Greven, A., den Hollander, F.: Branching random walk in random environment: phase transitions for local and global growth rates. Probab. Theory Related Fields 91 (1992), no. 2, 195-249.

[14] Griffeath, D.: The Binary Contact Path Process, Ann. Probab. 11 (1983), no.3, 692-705.

[15] Hu, Y., Yoshida, N. : Localization for Branching Random Walks in Random Environment, Stoch. Proc. Appl. 119, no.5, 1632-1651, (2009).

[16] Kesten, H.; Stigum, B. P. : A limit theorem for multidimensional Galton-Watson processes. Ann. Math. Statist. 37 (1966) 1211-1223.

[17] Lacoin, H. : New bounds for the free energy of directed polymers in dimensions $1+1$ and $1+2$, preprint.

[18] Liu, Q.; Watbled, F.: Exponential inequalities for martingales and asymptotic properties of the free energy of directed polymers in a random environment, preprint, 2008, to appear in Stoch. Proc. Appl.

[19] Nakashima, M: Central Limit Theorem for Branching Random Walk in Random Environment, preprint 2008.

[20] Révész, P. (1994): "Random Walks of Infinitely Many Particles" World Scientific.

[21] Rockafeller, R. T.: Convex Analysis, Princeton University Press, 1970.

[22] Smith, W. (1968): Necessary conditions for almost sure extinction of a branching process with random environment. Ann. Math. Statist. 39, 2136-2140.

[23] Smith, W., L.; Wilkinson, W., E. (1969): On branching processes in random environments. Ann. Math. Statist. 40 814-827. 
[24] Song, R. and Zhou, X. Y. : A remark on diffusion on directed polymers in random environment, J. Stat. Phys. 85, Nos.1/2, 277-289, (1996).

[25] Yoshida, N.: Central Limit Theorem for Branching Random Walk in Random Environment, Ann. Appl. Proba. 18 (2008) no. 4, 1619-1635.

[26] Yoshida, N.: Phase Transitions for the Growth Rate of Linear Stochastic Evolutions, J. Stat. Phys. 133, No.6, 1033-1058, (2008).

Francis Comets

Université Paris Diderot - Paris 7

Mathématiques, Case 7012, Bâtiment Chevaleret

75205 PARIS CEDEX 13

France

comets@math.jussieu.fr

Nobuo Yoshida

Division of Mathematics, Graduate School of Science,

Kyoto University,

Kyoto 606-8502,

Japan,

nobuo@math.kyoto-u.ac.jp 